\title{
A galaxy classification grid that better recognises early-type galaxy morphology
}

\author{
Alister W. Graham ^ \\ Centre for Astrophysics and Supercomputing, Swinburne University of Technology, Hawthorn, Victoria 3122, Australia.
}

Accepted 2019 June 7. Received 2019 June 7; in original form 2019 April 26

\begin{abstract}
A modified galaxy classification scheme for local galaxies is presented. It builds upon the Aitken-Jeans nebula sequence, by expanding the Jeans-Hubble tuning fork diagram, which itself contained key ingredients from Curtis and Reynolds. The two-dimensional grid of galaxy morphological types presented here, with elements from de Vaucouleurs' three-dimensional classification volume, has an increased emphasis on the often overlooked bars and continua of disc sizes in early-type galaxies - features not fully captured by past tuning forks, tridents, or combs. The grid encompasses nuclear discs in elliptical (E) galaxies, intermediate-scale discs in ellicular (ES) galaxies, and large-scale discs in lenticular (S0) galaxies, while the E4-E7 class is made redundant given that these galaxies are lenticular galaxies. Today, these structures continue to be neglected, or surprise researchers, perhaps partly due to our indoctrination to galaxy morphology through the tuning fork diagram. To better understand the current and proposed classification schemes - whose origins reside in solar/planetary formation models - a holistic overview is given. This provides due credit to some of the lesser known pioneers, presents some rationale for the grid, and reveals the incremental nature of, and some of the lesser known connections in, the field of galaxy morphology.
\end{abstract}

Key words: galaxies: elliptical and lenticular, $\mathrm{cD}$ — galaxies: general — galaxies: structure - history and philosophy of astronomy

\section{INTRODUCTION}

Clues to the past evolution of galaxies can be found encoded within their morphologies. The principal structures are the spheroid and disc, and the bar and spiral arms within the disc. A fundamental galaxy classification scheme should, therefore, capture these elements at some level, and may help to reveal links between observations and physical processes. For example, in the mid-1900s, an often heralded justification / success of the Jeans-Lundmark-Hubble galaxy sequence (Jeans 1919a; Lundmark 1925, 1927; Hubble 1926) was how it tracked the mean age of the host galaxies' stellar population.

Although the Jeans-Hubble tuning fork diagram (Jeans 1928, Hubble 1936) provided minimal information about the early-type galaxies (ETGs), the greater detail regarding the late-type galaxies (LTGs) was an invitation that theorists and modellers responded to (e.g. Roberts et al. 1975; Feitzinger \& Schmidt-Kaler 1980; Toomre 1981, and references therein; Haass et al. 1982). In contrast, the long thin handle of the "tuning fork" likely hindered an awareness as to the prevalence of substructures, such as discs and bars, within the ETG population (E, ES ${ }^{1}$, and $\left.\mathrm{S} 0\right)$. This situation

\footnotetext{
^ E-mail: AGraham@swin.edu.au

1 Bridging the elliptical (largely discless, except for an optional nuclear
}

was partly remedied by the Hubble-influenced galaxy classification schemes of de Vaucouleurs (1959a,b) and Sandage (1961), but the level of complexity in their schemes may have inhibited a more wide-spread uptake.

The new galaxy morphology classification grid shown herein, stemmed from the recent analyses of several ETGs with intermediate-scale discs. This started with an investigation into the remarkably boxy, dwarf ETG known as LEDA 074886 (Graham et al. 2012). This galaxy harbours an edge-on, intermediate-scale disc - whose rotation was revealed using the Keck Telescope - and it was unclear where such a galaxy resides in the galaxy classification schemes. It was similarly problematic trying to place the dwarf ES galaxy CG 611 (Graham et al. 2017, see their section 3.3.2) into the spin-ellipticity diagram of slow and fast rotators (Emsellem et al. 2007, 2011). In this galaxy, the intermediate-scale disc appears face-on, revealing the presence of a bar. Such fully-embedded discs

disc) and lenticular galaxies (with large-scale discs dominating the light at large radii), Liller (1966) identified the ES galaxy type with intermediatescale discs (e.g. Capaccioli \& Vietri 1988; Michard \& Simien 1988; Nieto et al. 1988, 1991; Simien \& Michard 1990; Michard \& Marchal 1993). Following Liller, who concatenated the letters E and S0 to give the ES galaxy type, Graham et al. (2016) concatenated the words elliptical and lenticular, to give the "ellicular" galaxy name. 
are not confined to dwarf ETGs. For example, Section 3.3 of Graham et al. (2016) reports on the massive ES galaxy NGC 1271 in the Perseus cluster. Additional recent examples of ordinary ETGs with intermediate-scale discs can be seen in Savorgnan \& Graham (2016b) and Sahu et al. (2019).

The ETGs have long been a misclassified, and consequently misunderstood, population. Indeed, the discs of lenticular galaxies were not originally recognised as such, but rather they were regarded as demarking the "fundamental plane" of lentil-shaped galaxies. Furthermore, unless these discs were orientated rather edge-on to our line-of-sight, or contained circular dust lanes/rings, then they usually went undetected. As noted by Capaccioli (1990), most ETGs contain a large-scale disc, and many of these discs contain a bar, while some also contain ansae (Martinez-Valpuesta et al. 2007) at the ends of the bar, and rings. Studying galaxy images, Rix \& White (1990) reported that almost all non-boxy ${ }^{2}$ "elliptical" galaxies could have discs, and studying the kinematics of bright elliptical galaxies, Graham et al. (1998) highlighted that the actual number of dynamically hot ${ }^{3}$ stellar systems is much lower than previously thought. Weak bars have also tended to be overlooked, as noted by Gutiérrez et al. (2011) and found by Sahu et al. (2019). Some of the general ongoing confusion surrounding the ETG population, arising from their treatment as discless, one-component systems, may, in part, originate from their inadequate representation in the tuning fork diagram that we were all undoubtedly introduced to through countless good textbooks and online resources.

In order to develop or modify a galaxy classification scheme that more fully captures the morphology of ETGs, the origins of, and many subsequent variations to, the tuning fork diagram are reviewed in Sections 2 and 3, respectively. What becomes apparent is the incremental nature of progress, and the continual re-working of particular themes. In that regard, this paper is no different. Section 4 presents a diagram, with a grid structure that better represents the ETGs. The new feature of this grid is the inclusion of Martha Liller's ES galaxies, capturing the range of disc sizes in ETGs. Although known for half a century (Liller 1966; di Tullio 1978), the intermediate-scale discs of ES galaxies have repeatedly been overlooked.

It is hoped that the grid will help facilitate a greater awareness of the primary structures in ETGs, which are still not immediately recognised in some galaxy research today. Section 5 includes a discussion of possible evolutionary paths for ETGs within the grid, points out connections with kinematic classifications, and provides an outlook into large automated surveys in which the capacity of human classifiers to assign a morphological type has been surpassed.

\section{HISTORICAL BRIEFING}

In an effort to distill the patterns seen in the nebula catalogs of $\mathrm{La}$ Caille (1755) and Messier (1781), and of course the larger imaging campaigns of both the Herschel family $(1786,1864)$ and Parsons $^{4}(1878)$ - which led to the New General Catalogue (NGC:

\footnotetext{
2 The term "boxy" refers to galaxies whose isophotal shapes are slightly more rectangular than elliptical.

3 "Dynamically hot" refers to a system where random (stellar) motion dominates over ordered (stellar) motion, i.e. where a galaxy's velocity dispersion dominates over the rotational support.

4 William Parsons, the $3^{\text {rd }}$ Earl of Rosse, discovered the spiral nebulae (Rosse 1850a,b).
}

Dreyer 1888) and the supplementary Index Catalogue (IC: Dreyer 1895) - new classification schemes were explored by Wolf (1908), Knox Shaw (1915), Curtis (1918), and others. Collectively, this contributed towards the Jeans $(1919 \mathrm{a}, \mathrm{b})$ evolution sequence for galaxies, which drew direct analogy from the Laplace (1796, 17991825) model $^{5}$ of planetary formation, in which a cooling and thus contracting ${ }^{6}$ nebula forms a rotationally-induced equatorial bulge ${ }^{7}$, before throwing off rings ${ }^{8}$ of matter, into the equatorial plane, out of which planets subsequently condense.

Following ongoing works pertaining to solar system formation (e.g. Tisserand 1889-1896; Keeler 1900, his p.348; Chamberlin 1901; Moulton 1905; and Aitken 1906, see his p.118-119), Jeans $(1919 a, b)$ embraced the idea that as a nebula ages, it will form a lenticular shape due to its increased rotation. He similarly embraced the Aitken (1906) modification to the Laplace model, in which an external gravitational force from a passing nebula will invoke the formation of symmetric spiral arms — often modelled as "equiangular" spirals, i.e. logarithmic spirals (von der Pahlen 1911; Groot 1925; Davis et al. 2017) — rather than rings. It was speculated that this external gravitational perturbation would result in a system-wide tide - akin to the Moon-induced tides on either side of the Earth - sufficient for the tidal theory of Roche (1850) to generate near- and far-side protrusions from the nebula that eventually lead to spiral arms because of the system's rotation (Aitken 1906; see also Alexander 1852). These arms would grow out of the (shrinking) nebula's material, and move outward with time, becoming more open and eventually producing (gravitational) condensations which, in the galaxy scenario, are not planets but star clusters. ${ }^{9}$ The Laplace-Aitken model for solar nebulae is the basis of the Jeans (galaxy) "nebula hypothesis" for a sequence from smooth early-type (young) elliptically-shaped nebulae progressing toward lenticular nebulae, then early-type spirals with prominent lenticular-shaped bulges, to late-type (old) spiral nebulae, which have small bulges, are increasingly complex in appearance, and have open spiral arms and increasingly noticeable condensations of stars in these arms. This galaxy evolution sequence was popularly discussed in the early 1920s (e.g. Reynolds 1921, 1925). The spheroid or central bulge, and the spiral arms, are primary elements of this scheme. Reynolds (1925, see his p.1016) also remarks that a limited number of edge-on lenticular nebula, e.g. NGC 5868 and NGC 2859, were already known at that time, bridging the elliptical and spiral nebulae.

Supplanting the classification proposed by Hubble (1922), Hubble $(1926)^{10}$ adopted this broad sequence from Jeans (1919a,b),

\footnotetext{
5 Prentice (1978, 1984; see also Prentice \& Dyt 2003) developed the modern Laplacian theory. Today, there is competition from the solar nebular disc model (Safronov 1969).

${ }^{6}$ The collapse is described by the Jeans (1902) instability that was advanced to explore the "nebular hypothesis" of Swedenborg (1734), Kant (1755), and Laplace (1796, 1799-1825).

7 See also Gott (1975), Freeman (1975), and Larson (1975) for related models.

8 Only rings, not spirals, are seen in the early, nebulae catalogue drawings (e.g. Herschel 1833, his Figure 25). As such, the Laplace (1825) model involves the generation of rings rather than spirals.

9 In the early 1900s, before the "island universe" nature of the distant "nebulae" were known, some astronomers thought that the spiral nebulae, which we now know are spiral galaxies, were solar systems in formation (e.g. Sutherland 1911, but see Schwarzschild 1913).

${ }^{10}$ Hubble (1926) built on a manuscript that Edwin Powell Hubble sent to Vesto Melvin Slipher in 1923 for circulation to the members of Commission 28, on Nebulae, at the upcoming second I.A.U. meeting to be held in 1925.
} 


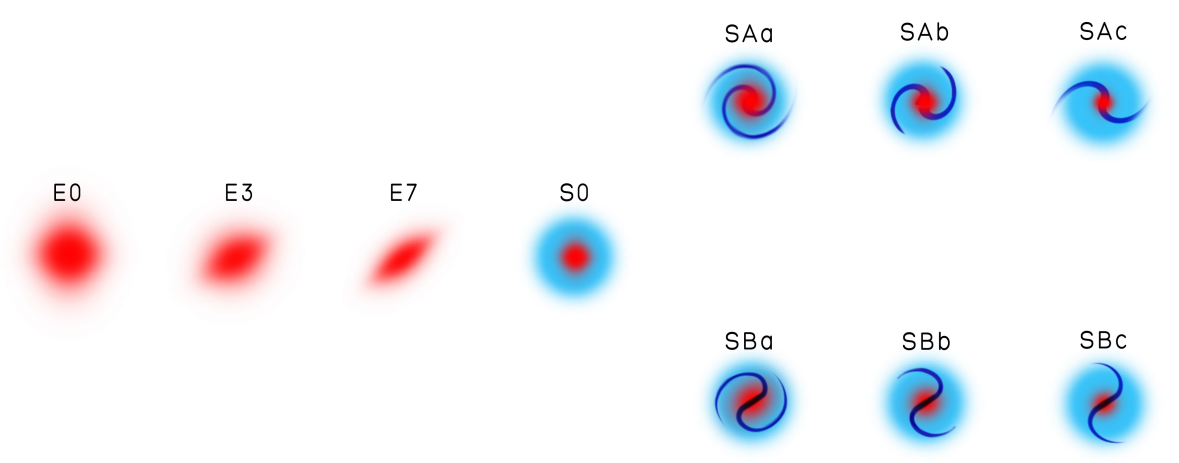

Figure 1. Jeans-Hubble tuning fork (Jeans 1928; Hubble 1936), including the S0 galaxy morphological type theorised in Jeans (1919a), identified in Reynolds (1925, see his p.1016), and later added by Hubble (1936).

while also incorporating the spiral sequence from Reynolds (1920). However, by the mid-1920s most papers were dropping the idea of a temporal sequence, and Hubble (1926, footnote 1 of p.326) also now distanced himself from the temporal connotation which he had initially built into his classification scheme through his use of the words "early" and "late". ${ }^{11}$ While Hubble (1926) now embraced only the morphological sequence of Jeans and not the evolutionary sequence $^{12}$, in mimicking Jeans, Hubble (1926) had, somewhat ambiguously, written that "the arms appear to build up at the expense of the nuclear regions and unwind as they grow; in the end, the arms are wide open [highly resolved] and the nuclei inconspicuous".

The first criteria used by Hubble (1926) was the relative size of the unresolved nuclear region, i.e. the bulge or spheroid. Then followed the ellipticity in the case of the ETGs, and for the LTGs it was the degree of resolution in the spiral arms, and the extent to which they are unwound. Interestingly, Lundmark $(1925,1927)$ used concentration, rather than ellipticity, to classify the then-called globular nebulae, i.e. the elliptical objects, in his scheme. As such, while Hubble (1926) focussed on the progression of forms (ellipticity, spiral arms), Lundmark had inadvertantly hit upon what we now know is a concentration-mass sequence among the ETGs ${ }^{13}$ (e.g. Davies et al. 1988; Caon et al. 1993). Shapley (1927) and Shapley \& Ames (1929) would subsequently use both concentration and elongation of form in their Virgo-Coma galaxy catalog.

Hubble (1926) additionally included a parallel sequence for the non-barred (Normal) and barred ( $\phi$-type) spiral nebulae that had been pointed out by Knox-Shaw (1915) and Curtis (1918, his page 12). While undoubtedly something of a cut-and-paste job, Hubble's classification scheme based on his summary of appropriate pieces of information - which is often how science progresses - resulted in a concise and much used synopsis of extragalactic anatomy. The classification summarised in Hubble (1926) included Elliptical (E0-E7), Normal, i.e. non-barred, Spiral ( $\mathrm{Sa}, \mathrm{Sb}$, $\mathrm{Sc}$ ), Barred Spiral (SBa, SBb, SBc), and Irregular (Irr) nebulae.

11 In Hubble's initial manuscript, which he sent to Slipher in 1923, he wrote that "there is some justification in considering the elliptical nebulae as representing an earlier stage of evolution", and in 1923 he also now listed them before the spiral nebulae (cf. Hubble 1922, his p.168; see also the detailed review by Hart \& Berendzen 1971, their p.114).

12 Block \& Freeman (2009) discuss how Hubble sought out Reynolds help, apparently prior to 1926 , to develop a classification scheme that would be comparable to the Jeans' evolution sequence. Jeans (1931) subtly notes that Hubble succeeded.

13 Lundmark (1925) also used concentration to separate the nebulae within the spiral class.
Reynolds (1927) called Hubble's attention to pre-existing and rather similar classification schemes which were not cited in Hubble (1926). This followed Hubble (1926, footnote 2 of p.323) which called-out Lundmark (1925, see his p.867) for having presented a "practically identical" classification scheme but having not cited Hubble. Lundmark had, however, established his classification scheme by 1922 , before Hubble sent his initial manuscript to Slipher in 1923 (see Teerikorpi 1989, and page 24 in Lundmark 1927). Reynolds was also likely motivated to act as the ethical police given that Hubble (1923) had found that Reynolds' (1913, 1916, 1920) model for the bulges of spiral nebulae, also described elliptical nebulae. Yet Hubble (1923) gave no credit to Reynolds, whose model subsequently became known as the Hubble law, or occasionally the Hubble-Reynolds law, not to be confused with the Hubble-Lemaître law connecting galaxy distances with redshifts — something which was also previously addressed ${ }^{14}$ by Lundmark (1925).

As Block et al. (2004) reminded us, it was actually Sir James Jeans (1928) who came up with the Y-shaped diagram that encapsulated this early-to-late type galaxy sequence that was later turned sideways and became known as the Hubble tuning fork (Figure 1). Rather than a single linear sequence, the two arms of the Y-shaped figure were invoked to capture the spiral nebulae that Curtis (1918) had noted come both with and without bars. In Hubble's (1936) version of this Y-shaped diagram, he added the lenticular (S0) galaxy type from Reynolds (1925) — the so-called "armless spiral" galaxies, if ever there was an oxymoron - at the nexus point. Including this previously hypothetical galaxy type from Jeans (1919a,b), Hubble (1936) expanded upon Hubble (1926) by expressing an awareness of the continuity of forms among the entire (luminous) galaxy population, as opposed to an elliptical branch versus two spiral branches. However, the variation in ETG morphology was not yet fully appreciated at this time.

14 Building upon Fath (1909), Slipher $(1915,1917)$, and Campbell \& Paddock (1918) who had identified NGC 4151 as a spiral nebula with a redshifted velocity of $940 \mathrm{~km} \mathrm{~s}^{-1}$, Lundmark (1925) referred to these redshifts as "Campbell shifts", now known as "Hubble redshifts". Although nowadays very much in the shadow of Hubble (1929), Lundmark (1925, p.867) wrote that "A rather definite correlation is shown between apparent dimensions and radial velocity, in the sense that the smaller and presumably more distant spirals have the higher space-velocity", and he presented a second-order distance-velocity relation which effectively reduces to Hubble's (1929) first-order distance-velocity relation upon setting $m=0$. 


\section{REVISIONS, ADAPTIONS, ENHANCEMENTS}

Having a better understanding of the tuning fork diagram's origin, we are well-placed to now appreciate and understand the revisions and re-expressions that it has experienced over the years.

\section{1 de Vaucouleurs classification volume}

The tuning fork diagram has had a remarkable longevity, and witnessed a continual stream of proposed upgrades and complementary classification schemes. For example, Shapley \& Paraskevopoulos (1940) introduced the Sd galaxy type, and de Vaucouleurs (1959a) introduced the Magellanic-like Sm galaxy type, and also the Im galaxy type for Irregular (Irr) galaxies related to the Small Magellanic Cloud, which included both barred and unbarred versions. Around this time, Holmberg (1958) introduced the subdivisions, i.e. the Sab, Sbc galaxy types.

Working with Hubble's (unpublished) notes written prior to his death in 1953, de Vaucouleurs (1959a) points out that one of the main revisions that Hubble wanted to make to the galaxy classification system was the introduction of three groups of barred lenticular galaxies, defined largely according to the contrast and strength of the bar. This was captured by de Vaucouleurs (1959a, 1959b, see his Figures 9 and 11), which introduced the weak/anemic bar class $(\mathrm{AB})$ for both spiral and lenticular galaxies, slotting in between galaxies with a clear bar (designated by the letter B) and those without (designated by the letter A). de Vaucouleurs (1959a) also replaced the phrase, "normal spiral" with "ordinary spiral", to reflect the fact that comparable numbers of barred and non-barred spiral galaxies were observed, and thus one type was not more normal than the other.

These additions can be thought of as converting the tuning fork into a trident, with a new middle prong for the $\mathrm{AB}$ types having weak bars. However, de Vaucouleurs $(1959 a, b)$ stepped things up yet another level, and introduced not just three parallel sequences (A, AB, B) for the S0 and spiral galaxies, but also an orthogonal third axis to the galaxy classification scheme in order to designate whether the spiral arms ${ }^{15}$ originate from a ring or not $(\mathrm{r}=$ ring,

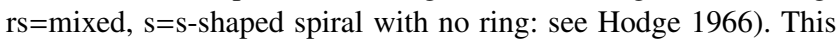
culminated in a three-dimensional classification volume, which was further embellished with additional morphological details, which can be appreciated from the extensive classification types in the Third Reference Catalog of Bright Galaxies (de Vaucouleurs et al. 1991).

Arguably, the three-dimensional classification volume of de Vaucouleurs (1959a) departed too far from the simplicity of the two-dimensional tuning fork. Indeed, there were three stages, from early- to late-type, just within the lenticular galaxy class $\left(\mathrm{S}^{-}, \mathrm{S}^{0}\right.$, $\left.\mathrm{S}^{+}\right)$; this was in addition to the $\mathrm{E}^{+}$stage bridging the $\mathrm{E}$ and $\mathrm{S} 0$ galaxies, and the S0/a stage bridging the $\mathrm{S} 0$ and Sa galaxies. The various bells and whistles added by de Vaucouleurs resulted in a classification scheme approaching the complexity of that introduced by W.Herschel (Curtis 1933, see p.919) or the 5-number sequence introduced by his son J.Herschel (Lundmark 1927, p.19). This level of complexity reflected a recognition that ETGs are not

15 Danver (1942) predicted that a classification of just the arm patterns may one day be needed, effectively echoing Reynolds (1927) criticism of the simplified system of Hubble (1926) which overlooked the different character of the spiral arms. Four decades later, Elmegreen \& Elmegreen (1982) brought in a fine microscope and introduced 12 distinct arm classes. simple, single-component systems. However, in spite of de Vaucouleurs classification volume, many research papers continue to flounder by treating ETGs as though they are single component systems. This may in some instances arise from recourse to the simplicity of the tuning fork.

\subsection{Sandage's Hubble Atlas}

Sandage, Hubble's former $\mathrm{PhD}$ student who had taken possession of Hubble's notes and loaned them to de Vaucouleurs in the mid 1950s, used these to produce his own "Hubble Atlas of Galaxies" (Sandage 1961; see also Sandage 1975). The properties of the spiral arms - such as their winding angle, the degree of condensation — rather than the apparent bulge-to-total $(B / T)$ flux ratio, were now made the primary criteria in establishing the spiral morphological types. In a second cautious distinction from Hubble (1926), Sandage (1961, page 6) suggested that the Aitken-JeansLundmark-Hubble sequence was an evolutionary one, such that galaxies started out as "late-type spirals" with young stars that evolved into "early-type spirals" with old stars that subsequently morphed into "early-type galaxies", but he clearly noted that it was an unproven idea. His motivation came from the known trends involving the stellar populations (e.g. Morgan \& Mayall 1957; Morgan 1958).

As noted by Sandage (1961), disc galaxies of a given Hubble type had a range of $B / T$ flux ratios. This is because, as just noted, the first criteria used in Sandage's classification scheme was the openness of the spiral arms, followed by the resolution of the arms into stars. Progressing from ETGs which do not have spiral arms, to the Sa galaxies having tightly wound spiral arms with pitch angles of a few to $\sim 10$ degrees, to the late-type spirals with open arms and pitch angles of up to $\sim 40$ degrees, there is a sequence. The existence of some S0 galaxies with a low $B / T$ flux ratio is still consistent with the picture that the $\mathrm{S} 0$ galaxies are a transition class between the $\mathrm{E}$ and Sa galaxy types. However, this breaks down somewhat in schemes using the $B / T$ flux ratio as the primary diagnostic while the nature of the spiral arms takes second-place.

No doubt in part due to the greater ease in estimating $B / T$ flux ratios than spiral arm winding angles and resolution (which can be impeded by the disc inclination to our line-of-sight and image depth), modern classifications have a tendency to follow Hubble's and de Vaucouleurs' ordering of the criteria and align themselves more with the $B / T$ flux ratio than with the spiral arms. Today, each spiral galaxy type, and the $\mathrm{S} 0$ galaxy type (which can contain rings, i.e. the most tightly wound of all spiral arms), do still possess a range of $B / T$ flux ratios (e.g. Freeman 1970; Boroson 1981; Kent 1985; Kodaira et al. 1986; Simien \& de Vaucouleurs 1986; Graham \& Worley 2008; Laurikainen et al. 2010). This is in part because different classifiers have used alternating primary and secondary criteria to classify galaxies.

Sandage (1961; see also Figure 75 in Bigay 1963) also had three subgroups of S0 galaxy, depending on the degree and location of dust lanes, plus three subtypes of barred S0 galaxy, depending on the nature of the bar. Indeed, Sandage, de Vaucouleurs, and Hubble, considered bars to be a primary criteria in their classification schemes.

\subsubsection{Bars}

While Jeans (1902) explored the stability and balance (in the earlystage of spherical nebulae) between the outward pressure of a hot 
gas cloud and the inward force of gravity, Safronov (1960; see also Gurevich \& Lebedinsky 1950) additionally considered the shear forces arising from the differential (Keplerian) rotation within the discs of both proto-planetary clouds and the Milky Way. Toomre (1964), in collaboration with Agris Kalnajs, expanded this to the discs of external galaxies. In addition to Hunter (1963), who explored disc stability using oblate spheroidal coordinates and solutions to Laplace's equation, Safronov and Toomre established idealised stability criteria which could lead to the coagulation of discs and also the formation of bars (Hohl 1971). While subsequent work including dark matter halos offered a stabilising environment for discs, bars do still form from these global disc instabilities in which the circular disc orbits become elongated to form the bar (e.g. Combes \& Sanders 1981; Combes \& Elmegreen 1993; Sellwood \& Wilkinson 1993; Athanassoula 2003). Bars can also experience instabilities of their own, resulting in a buckling both within and above the disc plane (Combes et al. 1990; Athanassoula 2005; Saha et al. 2018) to produce X/boxy/(peanut shell)-shaped "pseudobulge" structures or "barlenses" which were added to the expanded galaxy classification scheme of Buta et al. (2010).

Bars themselves are also a recognised driving force in secular galaxy evolution. Given how the dynamical (in)stability of the disc can be seen through the appearance of bars, the tuning fork diagram, plus the extension to include weak bars, reflects an important physical process. Bars may also offer insight into the triaxiality, central density and rotation of the dark matter halos thought to surround galaxies (e.g. Debattista \& Sellwood 2000; Athanassoula 2002, 2003; Berentzen et al. 2006). It is therefore considered desirable to retain the bar strength in a schematic of galaxy classification.

\subsection{Yerkes system}

The Yerkes system of galaxy classification used the radial concentration of light, as suggested by Lundmark (1925), plus optical spectra to trace the stellar populations (Humason et al. 1956; Morgan \& Mayall 1957; Morgan 1958, 1959; Morgan \& Osterbrock 1969). For luminous galaxies, the subjectively-defined concentration parameter set (denoted: a, af, f, fg, g, gk, k) helped distinguish between the relative prominence of the bulge and disc, and it reportedly correlated better with the central stellar population than the Hubble morphological-type did. ${ }^{16}$ The Sérsic (1963) index $n$ (see Graham \& Driver 2005 for a review of the $R^{1 / n}$ model) was also designed to quantify the varying bulge-to-disc flux ratio, and Graham et al. (2001) showed that the galaxy Sérsic index is monotonically related to the galaxy's mean concentration index (Okamura et al. 1984). However, unlike with the Sersic index, this objectivelydefined concentration parameter is rather sensitive to the image exposure depth (Graham et al. 2001; see also Pović et al. 2015). Furthermore, dwarf ETGs have the same concentrations as latetype galaxies (LTGs), and thus a single concentration parameter does not define the ETG-to-LTG sequence if one wishes to include dwarf galaxies. While the inclusion of galaxy type, or rather "form

16 While the Yerkes system proposed replacing the $\mathrm{Sa}, \mathrm{Sab}, \mathrm{Sb}, \ldots$ sequence with the radial concentration of the light, it retained the four broad "form families" (Elliptical, Spiral, Barred Spiral, and Irregular) as a secondary parameter and added four more: dusty elliptical, nucleated, low surface brightness, and rotationally-symmetric but without clear elliptical or spiral structure. In addition, the axis-ratio was used to define a second secondary parameter, referred to as the "inclination class", which ranged from 1 (spherical) to 7 (elongated). family", within the Yerkes system resolves this ambiguity, it also highlights the value in retaining a morphological descriptor.

The central, (optical luminosity)-weighted spectra provided mean age estimates along the galaxy sequence, offering hope of unlocking the formation history of galaxies. It revealed an abundance of early-type stars in late-type galaxies, and late-type stars in earlytype galaxies. Although helpful, as can be inferred from van den Bergh (1975, his p.60-61), mass-weighted ages, or star-formation rate histories of the separate bulge and disc components, are more desirable and less prone to mis-direction. Studying spectra from the bulges of spiral galaxies, MacArthur et al. (2009) found that both early- and late-type spiral galaxies possess old mass-weighted ages, with less than one-quarter of the stellar mass arising from young stars. Variants of the Lundmark-Yerkes system are still used today, as seen in the concentration-colour plane for bright galaxies (e.g. Driver et al. 2006).

The cD galaxies (Matthews et al. 1964; Morgan \& Lesh 1965) of the Yerkes system are galaxies with three-dimensional shrouds, known as halos or envelopes, which belong more to the host galaxy's cluster than to the cluster's central cD galaxy. These galaxies are typically ETGs, and not some new kind of galaxy.

\subsection{The van den Bergh trident}

The David Dunlap Observatory (DDO) classification system for late-type galaxies (van den Bergh 1960a,b,c) introduced luminosity classes based on the correlation between the absolute magnitude of spiral galaxies and the degree to which their spiral arms are developed. It was later expanded to include dwarf galaxies (van den Bergh 1966). The DDO system was substantially re-worked by van den Bergh (1976), who now based it on disc galaxies with either: strong spiral arms, i.e. spiral galaxies (S); weak/anemic spiral arms, i.e. S0/a galaxies (A); or no spiral arms (S0 galaxies). This produced the three prongs of a trident whose handle was the E0-E6 sequence. Attention to the arm strength was favoured over the bar strength, and the principle axis of the disc galaxy sequence now reflected the bulge-to-total flux ratio (as in Hubble 1926) rather than the nature, i.e. the winding angle and condensations, of the spiral arms (as in the original DDO system and Sandage 1961). As such, the presence of bars was now overlooked in not only the ETGs but also the LTGs. However, the three prongs served to capture a continuity in spiral arm contrast that mimicked the varying gas content and luminosity-weighted stellar ages. Despite the substantial changes, van den Bergh referred to this as the Revised David Dunlap Observatory (RDDO) system.

The motivation behind this change was to reflect the galaxy formation scenario of Spitzer \& Baade (1951) rather than Jeans (1919a). Building on (i) the idea in Spitzer \& Baade (1951) that S0 galaxies are gas-stripped spiral galaxies, which have subsequently lost their spiral pattern, due to the environment of a galaxy cluster, and (ii) inspired by Sandage, Freeman \& Stokes (1970, see also Rood \& Baum 1967) who remarked that S0 and spiral galaxies have the same distribution of axial ratios, van den Bergh (1976) embraced the notion of S0 galaxies as a parallel sequence to spiral galaxies. This was in contrast to the notion that they are a bridging sequence between elliptical and spiral galaxies. However, such an origin from spiral galaxies, or at least a universal origin, was later ruled out because many $\mathrm{S} 0$ galaxies are more luminous than spiral galaxies (e.g. Burstein et al. 2005), and therefore alternative formation mechanisms are required for $\mathrm{S} 0$ galaxies. Furthermore, S0 galaxies do not just reside in clusters. Therefore, at least some are not stripped spiral galaxies. Indeed, ETGs with the same disc-like 
photometric and kinematic properties as those in galaxy clusters are known to exist in isolation (Janz et al. 2017; Graham et al. 2017).

Aside from the above issues negating the motivation for this trident, another obvious setback with the proposal for a true "parallel sequence" (among the prongs) based on the bulge-to-disc flux ratio is that the spiral galaxies are not structurally similar to ETGs with discs. That is, spiral galaxies do not have the high bulge-todisc ratios of some ETGs, and in general ETGs galaxies do not have the low bulge-to-disc ratios of many late-type galaxies (e.g. Graham \& Worley 2008). For example, ETGs can have discs fully contained within their spheroidal structures, they are neither E nor S0 galaxies, while many late-type galaxies can be bulgeless but an abundance of such S0 counterparts are not known. The markedly different histogram of bulge-to-total flux ratios between ETGs with discs and the spiral morphological sequence was shown by Freeman (1970, his Figure 9). Graham et al. (2016, their Figure 7) provides an expanded representation of this histogram sequence, better depicting the bulge-to-total flux ratio for early- and late-type galaxies. It shows the distinction between the bulge-dominated ES galaxies, S0 galaxies with both significant large-scale discs and low bulge-to-total flux ratios, and the tail in the distribution to bulgeless spiral galaxies. This highlights the incomplete nature of the prongs in the trident, i.e. that there is not a parallel sequence but rather only partial overlap which superficially appears substantial if one includes (with no number-density weighting) the underpopulatedwings of the bulge-to-total distribution from each morphological type.

Graham \& Worley (2008) show that S0 galaxies with low bulge-to-total $(B / T)$ flux ratios are relatively rare, yet they should not be if they formed from the gas-stripping of spiral galaxies. This is because late-type spiral galaxies are less massive than the earlytype spiral galaxies - remember the DDO system (van den Bergh (1960c) — and have lower stellar densities in their discs (e.g. Graham 2001, his Figure 3). Therefore, they should be easier to convert into gas-free, and eventually spiral-less, S0 galaxies than it is to transform the early-type spiral galaxies with their higher $B / T$ flux ratios.

Given all of the above factors, there is a preference for (full) parallel sequences involving bar strength, rather tnan (partial) parallel sequences involving spiral arm strength.

Rather than building S0 galaxies by stripping the discs of spiral galaxies, it may be that S0 galaxies are formed by accretion and the building of discs (e.g. Young et al. 2008; Kannappan et al. 2009; Wei et al. 2010; Alatalo et al. 2013; Moffett 2014; Graham et al. 2015, and references therein; Mahajan et al. 2018). This latter process is truncated in galaxy clusters, perhaps contributing to the relatively low numbers of spiral galaxies in clusters when compared to the field population.

\subsubsection{Further developments}

Modelling bars as separate components, and using the Sérsic (1963) $R^{1 / n}$ model for the bulge component, the decomposition of ETG images by Laurikainen et al. (2010) confirmed that most ordinary ETGs are disc-dominated, and it revealed a wide range of $B / T$ flux ratios that was also observed by Krajnović et al. (2013). This range of $B / T$ flux ratios motivated Cappellari et al. (2011) to adopt the trident classification of van den Bergh (1976), although they drew his trident slightly differently, re-aligning the location of the trident's handle (E0 to E5 in their scheme) with the trident's outer "lenticular galaxy" prong, and declaring a new paradigm for ETGs which they called the ATLAS ${ }^{3 D}$ comb. In addition, the degree of rotational support now supplanted the $B / T$ ratio along the primary axis of the $\operatorname{ATLAS}^{3 D}$ comb (Cappellari et al. 2011, their Figure 2), thereby embracing the scheme discussed by Bender (1988), Capaccioli \& Caon (1992), Kormendy \& Bender (1996) and others based on a galaxy's angular momentum.

While Cappellari et al. (2011, their Figure 1) note that the spiral galaxies do not actually have the same high $B / T$ flux ratios as the ETGs, they did use NGC 4452 - whose thin inner disc/bar is prominent at optical wavelengths due to its edge-on orientation to suggest that ETGs may have $B / T$ ratios as low as late-type spiral galaxies. However, due to its edge-on orientation, it is unknown what type of galaxy NGC 4452 is, as with IC 335 and the Spindle Galaxy NGC 5866. ${ }^{17}$ Simulations show that spiral galaxies, when viewed edge-on, can look like NGC 4452 (e.g. Valentini et al. 2017, their Figure 6). Moreover, such potential S0 galaxies are not only rare, but one may ask, Where are the face-on ETGs with small or no bulge ${ }^{18}$

Cappellari et al. (2011) suggested that the anemic spiral galaxies - midway between the S0 and strong spiral galaxies - have small amounts of gas and a passively evolving stellar population, and they associated these with the "red spirals" (e.g. Masters et al. 2010). However, Cortese (2012) subsequently revealed that these red spiral galaxies have star formation rates on par with ordinary spiral galaxies, undermining the suggestion that the "red spirals" are a bridging population in terms of reduced amounts of star formation activity, and issuing a warning for classifications schemes based on optical colour. Furthermore, the ETGs (with $M_{B}<-14 \mathrm{mag}$ ) also have a range of colours, and a range of masses, voiding the notion that either colour or mass might monotonically vary across the trident/comb. The blue, low-mass ETGs illustrate this point well (Driver et al. 2006; Lee et al. 2006; Mei et al. 2006; Deng et al. 2009; Schawinski et al. 2009; Kaviraj et al. 2011; George \& Zingade 2015; Graham et al. 2017). Nonetheless, for non-dwarf galaxies, there are some trends and partial parallel sequences within the trident/comb, in which for a given $B / T$ flux ratio, a range of disc galaxy types may be found.

Kormendy \& Bender (2012) amended their modified tuning fork from Kormendy \& Bender (1996, which included three modifications taken from de Vaucouleurs 1959a, and which is discussed next), to present a somewhat similar scheme to van den Bergh's trident and the $\operatorname{ATLAS}^{3 D}$ comb. However, Kormendy \& Bender (2012) placed the dwarf "elliptical" galaxies — which they called dwarf spheroidal galaxies in order to distance them from ordinary ETGs on the left of the diagram - next to the Irregular Magellanic type galaxies at the far right of the diagram. Graham (2019) explains in some detail the false dichotomy between dwarf and ordinary ETGs that was based on bends at $M_{B} \approx-18$ mag in structural parameter diagrams involving the arbitrary $50 \%$ radius, $R_{\mathrm{e}}$, and the

\footnotetext{
17 Measurements of the health (strength and thinness) of such discs, reflecting a lack of heating, i.e. movement of stars out of the disc plane, or a lack of significant accretion (e.g. Brook et al. 2004), are impossible to make for most galaxies and thus not incorporated into morphological classification schemes.

18 Kormendy \& Bender (2009) provide a 5-component fit to the major-axis of NGC 4452, with the bar and barlens inverted, and report a very small $B / T$ ratio of $\sim 0.02$. A similar edge-on galaxy is NGC 4762 (PGC 043733: van den Bergh 1976; Baillard et al. 2011; Jarrett et al. 2003), which has had its (geometric mean)-axis light profile modelled by Sahu et al. (2019) and has a $B / T$ flux ratio of 0.08 , on par with Sc galaxies (Graham \& Worley 2008, their Table 4). For comparison, Sdm/Sm galaxies tend to have ratios 2 to 3 times smaller, or no bulge (e.g. Walcher et al. 2005).
} 
associated surface brightness terms. Use of radii containing different percentages of a galaxy's light reveals how the location of the bends change by more than 3 mag, and thus the bend mid-point and the separation of dwarf and ordinary ETGs has nothing to do with galaxy formation processes but instead depends on the arbitrary definition of galaxy radii. Related calls for a division, or continuity, among the ETG population are also addressed in Graham (2019).

\subsection{Isophotal shape}

Using a Fourier analysis to quantify the departure of galaxy isophotes from pure ellipses, in an effort to detect photometrically weak discs via the presence of pointy/lenticular-shaped isophotes, as previously done by Carter $(1978,1987)$ and Cawson (1983), Bender (1988) wrote that "It is most curious that there is little evidence for significant morphological difference between rapidly and slowly rotating ellipticals". The answer to this curiosity is that the observed (on the plane of the sky) ellipticity of a disc varies with $\cos (i)$, while the kinematic contribution from the disc is measured perpendicular to the plane-of-the-sky, parallel to our line-of-sight, and varies with $\sin (i)$, where the disc's angle of inclination $i$ equals 0 degrees when seen face-on. Tables of $\cos (i)$ and $\sin (i)$ will immediately reveal why the presence of a disc is more apparent in the kinematics than in an image. Indeed, this justifiably led Cappellari et al. (2011) to prefer the use of kinematic measures rather than isophotal shapes for their ATLAS ${ }^{3 D}$ comb.

Bender (1988; see also Nieto et al. 1988) investigated the relation between the mean isophotal shape, i.e. boxy or discy, and the ratio of the inner (typically $\lesssim 1 R_{\mathrm{e}}$ ) stellar rotation and mean velocity dispersion, $V / \sigma$. Bender (1988) suggested that elliptical galaxies can be separated into two classes: those that are more closely related to the S0 galaxies, such that they are rotationally flattened with weak discs and have discy isophotes; and those with boxy isophotes that are dynamically supported by anisotropy in their velocity dispersion. Kormendy \& Bender (1996) subsequently visualised this through their modified representation of the elliptical galaxies in the tuning fork, now based on boxy-to-discy isophotes rather than ellipticity. Although, they noted a number of complications, and two others are mentioned here.

Kormendy \& Bender (1996) reported that the measured velocity anisotropy correlated with the mean isophotal shape and advocated for using the isophotal shape to represent the elliptical galaxies in the Jeans-Hubble tuning fork. However, Figure 2 in Kormendy \& Bender (1996), which plots isophotal shape versus $V / \sigma$, shows that galaxies with boxy isophotes have no correlation with $V / \sigma$. This may be a consequence of sampling the dynamics of both pure elliptical galaxies and massive S0 galaxies. Lenticular galaxies can have massive merger-built bulges — with partially-depleted cores created by coalescing supermassive black holes (Begelman et al. 1980) - and large-scale discs (e.g. Dullo \& Graham 2014). Their $B_{4}$ profiles ${ }^{19}$ vary with radius, changing from boxy to discy as the radius increases. An average inner isophotal shape does not capture the two-component nature of these galaxies.

Depending on the orientation of the disc to our line-of-sight, ES galaxies can have high ellipticities and discy-shaped isophotes at small radii - along with considerable rotational support within the apertures typically used to measure the kinematics — which

$19 B_{4}$ is the amplitude of the fourth-order Fourier cosine term used to describe variations from purely elliptical isophotes (e.g. Carter 1978; Ciambur 2015). then transition to low-ellipticities and elliptical, or even boxyshaped, isophotes at large radii (e.g. Nieto et al. 1991, their section 4.2). An edge-on example of this is LEDA 074886 (Graham et al. 2012; see also NGC 4638 in Ferrarese et al. 2006, their Figure 13), while a face-on example is LEDA 2108986 (CG 611: Graham et al. 2017). At least in the latter case, a fundamental formation mechanism is evident through the accretion of this ES galaxy's disc. In those ES galaxies where the kinematical measurements have been obtained at radii beyond the intermediate-scale disc, the rotation is observed to fall away (e.g. Arnold et al. 2014: Foster et al. 2016; Bellstedt et al. 2017; Rawlings et al. 2019).

A long string of papers have advocated for a divide between ETGs with boxy isophotes and a partially depleted core versus those with discy isophotes and either no depleted core or a central excess. ${ }^{20}$ However, such campaigns, which have effectively treated ETGs as single component systems, via a single $B_{4}$ parameter, have overlooked both the ES galaxy population bridging the E-to-S0 galaxies, and the massive lenticular galaxies noted above. The use of a single discy or boxy parameter, or indeed a single ellipticity parameter, can be misleading for ETGs. Classification based on the shape of the radial isophotal profile, rather than a single mean isophotal shape, would help when the discs are relatively edge-on. However, this approach will fail when the discs are relatively face-on. Classification by kinematics offers advantages here, but it is more expensive in terms of telescope time. For ETGs, a classification scheme recognising the continuum of disc extent relative to the extent of the spheroid (see Simien \& de Vaucouleurs 1986; Capaccioli et al. 1988) — obtained from an analysis and decomposition of the image - would be preferable to a single isophotal shape parameter which neither adequately captures the two-component (bulge/disc) nature of ETGs nor represents the radially varying kinematics and isophotal shapes in ES galaxies.

\subsection{Quantitative footings: CAS space}

The galaxy classification scheme of Lundmark $(1925,1927)$ involved the concentration ${ }^{21}$, and compressibility ${ }^{22}$, of galaxies within each of his four galaxy classes (ETG, LTG, Magellanic, Peculiar) discussed in the excellent review, at that time, of galaxies by Lundmark (1927). Lundmark's classes reflected the symmetry, or lack thereof, of the galaxies. The classification scheme of Hubble $(1926,1936)$ was also related to the concentration of the later type galaxies - as traced through the apparent bulge-to-disc flux ratio - in combination with the apparent smoothness of the ETGs versus the increasing condensations and clumpiness seen in the LTGs. Hubble (1936) has additionally noted how the galaxy's optical colour correlated with the morphological type.

Although the ellipticity parameter in Hubble's classification scheme can be more dependent on the viewing angle than the intrinsic major-to-minor axis of a galaxy, it offered an objective quantity rather than a subjective description. Not surprisingly, building on the Yerkes system, several objectively defined concentration parameters were later introduced, e.g. Fraser (1972, see also de Vau-

20 Jerjen \& Binggeli (1997) may have been the first to consider additional nuclear components in ETGs as defined relative to the galaxy's outer Sérsic profile; Graham et al. (2003) were the first to define and model the central deficit of light in ETGs relative to the inward extrapolation of the spheroid's outer Sérsic profile.

21 "The ratio of the nuclear light to the light of the outer portions".

22 Lundmark simply described this as "Different degrees of compressibility towards [the] galaxy centre". 
couleurs 1977) and Okamura et al. (1984, later popularised by Doi et al. 1992, 1993 and subsequently Abraham et al. 1994). As was recognised by Okamura et al. (1984), and later quantified by Graham et al. (2001), their mean concentration parameter is a rather strong function of the exposure depth, and, as such, it does not simply measure the intrinsic concentration of a galaxy's light. Trujillo et al. (2001) therefore introduced a new concentration parameter to minimise the influence of the galaxy exposure depth, and Okamura et al. (1984) had suggested that additional parameters, such as the mean surface brightness, could be introduced to try and reduce this observational bias and limitation of the mean concentration parameter.

Expanding on this push towards a parameterised version of the Jeans-Lundmark-Hubble galaxy classification scheme resulted in the introduction of (a)symmetry parameters to measure galaxy peculiarities and non-symmetries in the arms (e.g., Lindblad \& Elvius 1952; Schade et al. 1995; Rix \& Zaritsky 1995; Abraham et al. 1996; Conselice 1997) and the introduction of smoothness parameters to measure star-forming clumpiness or image "bumpiness" (e.g., Isserstedt \& Schindler 1986; Takamiya 1999; Conselice 2003; Blakeslee et al. 2006). Collectively, Concentration, Asymmetry, and Smoothness, have been used to produce a three-dimensional CAS galaxy classification scheme (Conselice 2003). Although not without its issues (e.g. Neichel et al. 2008; Huertas-Company et al. 2015), including image depth, spatial resolution, and galaxy orientation, it offers a helpful quantification of the properties used by Lundmark and Hubble.

In contrast with de Vaucouleurs' three-dimensional classification volume - with its axes of what is effectively bulge/disc ratio, bar strength and ring pattern - the CAS scheme reduces the emphasis on the disc instability that leads to the creation of bars (and the ensuing ansae and rings), in favour of better capturing merger activity and star-formation. This is increasingly important when probing higher redshifts (e.g. Griffiths et al. 1994; Driver et al. 1995; Giavalisco et al. 1996; Mortlock et al. 2013), where the Jeans-Lundmark-Hubble classification becomes less applicable due to the rising fraction of galaxies with Irregular structures. By increasing the emphasis on condensations, non-smoothness, asymmetries, and irregularities — which were already features of the Lundmark and Hubble classification schemes - the CAS scheme may better facilitate the identification of the physical processes which shaped galaxies, albeit at the expense of a helpful taxonomy for identifying distinct structural components of evolved galaxies.

Classifications based on various kinds of interactions have also been advanced (e.g. Vorontsov-Vel'Yaminov 1959), and an array of special symbols were used in the extensive Morphological Catalog of Galaxies (MCG: Vorontsov-Vel'Yaminov \& Arkhipova 1962-1974). Cross correlation with the classification of de Vaucouleurs (1959a,b) and Sandage (1961) can be found in VorontsovVel'Yaminov \& Noskova (1968). Not surprisingly, colour has also been used (e.g. Conselice 1997), although the presence of colour gradients in galaxies, and different colours for different components, plus the colour-magnitude relation for ETGs, and the above mentioned star-formation in "red spirals", complicates the derivation and limits the usefulness of a single optical colour parameter for galaxies.

Despite its merits, the CAS space still leaves want for a simple two-dimensional graphic which captures the primary morphology of established galaxies.

\section{A GALAXY MORPHOLOGY CLASSIFICATION GRID}

As noted earlier, for a long time "nebulae" were not known to contain spiral patterns, and therefore Laplace's (1796) nebular hypothesis did not generate spiral structures. However, after spiral nebulae were first observed by Parson in Rosse (1850), Aitken (1906) modified the Laplace model in an effort to explain these structures, and Jeans (1919a) subsequently embraced this scenario. When Hubble (1926) presented his classification scheme based upon the Jeans galaxy evolution sequence and the barred/non-barred spiral sequence of Reynolds (1920), it did not include lenticular S0 galaxies, but they were later added in Hubble (1936) once they had become more commonly known. When de Vaucouleurs (1959a) and Sandage (1961) presented their revised galaxy classification schemes, they did not mention ETGs with intermediate-scale discs. Such galaxies were not highlighted until Liller (1966). Although a spate of papers from 1988 to 1993 drew attention to this type of galaxy, to date, galaxy morphology diagrams remain somewhat lacking when it comes to this type of galaxy.

At different radii, individual ETGs can be both boxy and discy, a slow rotator and a fast rotator, in addition to possibly possessing a bar within a disc of differing radial extent. To help address this, Graham et al. (2017) presented a schematic of the typical bulgeto-disc flux ratio versus galaxy morphological type, revealing how the ES galaxies fit in. Figure 2 presents a complementary grid to better highlight the discs and bars in ETGs. There is an inherent usefulness in summarising the continuity of forms of today's galaxies into an easy-to-grasp (two-dimensional) diagram. Theories and simulations need to address when and how these forms arose (e.g. Fiacconi et al. 2015; Genel et al. 2015; Remus et al. 2015). Despite its critics, from Reynolds (1927) to Abraham (1996) and Conselice (2003), the Jeans-Lundmark-Hubble scheme went a long way to achieving this summary. By more fully populating the two-dimensional space (of form and bar strength) and switching from a tuning fork to a grid, greater information about the ETG population can be incorporated.

Figure 2 is something of a compromise between the simplicity of the tuning fork and the complexity of de Vaucouleurs' classification volume. The grid has effectively collapsed de Vaucouleurs (1959a) $\left(\mathrm{SO}^{-}, \mathrm{SO}^{0}, \mathrm{~S}^{+}\right)$series back down to the single $\mathrm{S} 0$ galaxy type included by Hubble (1936), and treated de Vaucouleurs (1959a) $\mathrm{E}^{+}$stage as though they are the ES type galaxies from Liller (1966). As such, the classifications shown in Figure 2 are familiar — albeit with the exception of the non-barred (EAS), weak-barred (EABS) and strong barred (EBS) continuity within the ES population - and the classifications can additionally be embellished with informative higher-order labels, as done by de Vaucouleurs (1959a,b), Buta et al. (2010), and Laurikainen et al. (2011) to denote the presence of components, such as rings and nucleation, that are increasingly modelled and quantified in modern galaxy decomposition work (e.g. Davis et al. 2019; Sahu et al. 2019).

As with ordinary ETGs, dwarf ETGs can also have spheroid/disc/bar/etc. components. They both occupy the left hand side of the grid, encompassing systems with a range of $B / T$ flux ratios but without a spiral density wave.

Two old caveats are noted. The perceived ellipticity of a Maclaurin (1742) ellipsoid or Jacobi (1834) triaxial structure can change with one's line-of-sight to the structure. Furthermore, due to the presence of intermediate- and large-scale discs, the ellipticity often changes with radius for the same line-of-sight to the galaxy. This should be kept in mind when viewing the handles of tuning forks, tridents, and the elliptical galaxy edge of the grid shown 


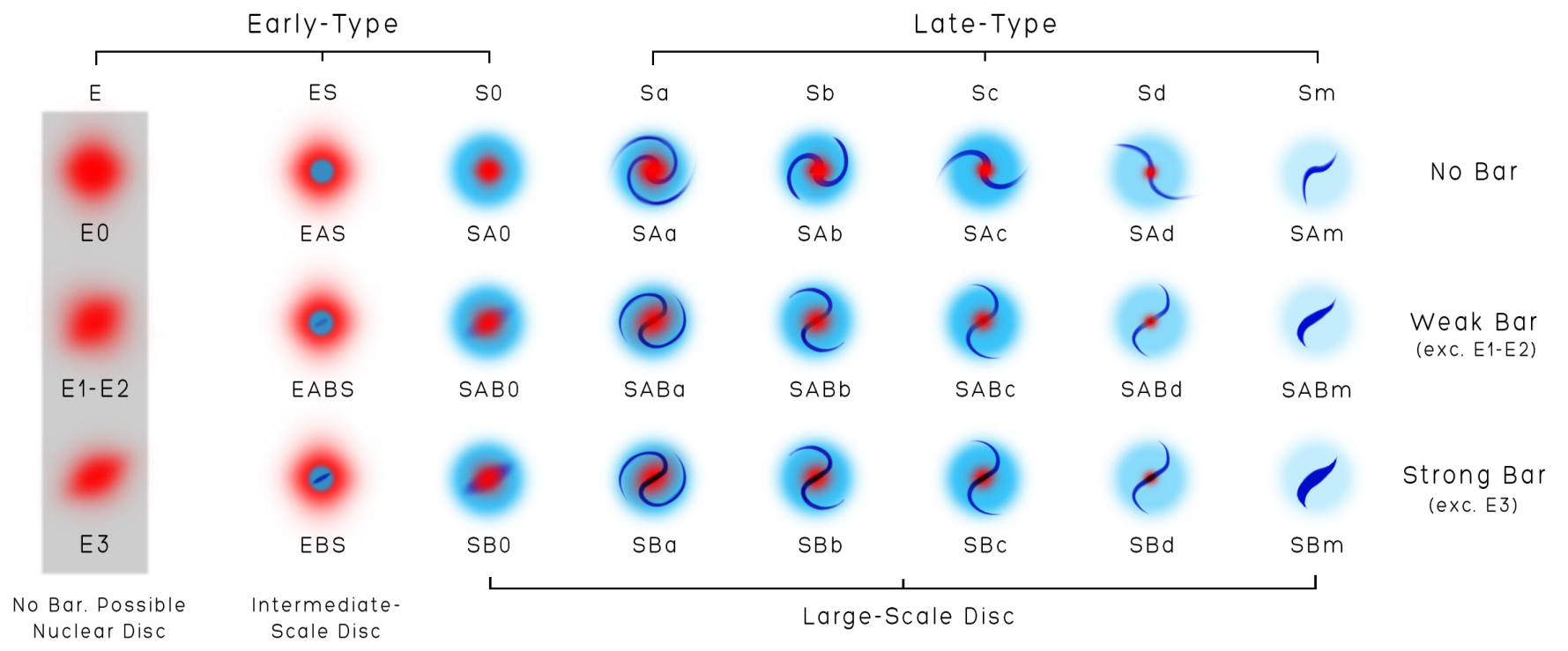

Figure 2. A two-dimensional grid of morphological types designed to provide a finer representation for the ETG population by better highlighting their discs and bars. There are direct parallels with the Jeans-Hubble tuning fork and de Vaucouleurs' (1959a) three-dimensional galaxy classification volume, especially if one considers de Vaucouleurs' $\mathrm{E}^{+}$galaxies to be the ES galaxies of Liller (1966). The relative prominence of the bulge and disc can be seen through the ETG section of the grid. The (largely) discless E0 to E3 sequence is slightly set aside in order to distance it from the bar designations on the right.

in Figure 2. Second, a galaxy's bulge-to-disc flux ratio and spiral arm pitch angle measurements can vary from author-to-author (due to methodologies, bandpass, measurement errors), and the image concentration depends on the observer's definition, exposure depth and measurement technique. Similarly, Lahav et al. (1995) reminded us that the assigned morphological type also varies from author-to-author. As such, there is a certain level of scatter along the principle-axis of the grid, whether it be defined primarily by the spiral arms, bulge-to-disc flux ratio, or the radial concentration of the light if excluding the dwarf galaxies.

While numerous additional schemes to morphologically classify galaxies have their merits (e.g. van den Bergh 1960c; Lekshmi et al. 2003; Lotz et al. 2004; Yamauchi et al. 2005; Conselice 2006; Scarlata et al. 2007; Vika et al. 2015; Ferrari et al. 2015; Selim \& Abd El Aziz 2017), it is hoped that the simplicity of, and continuity within, the grid shown in Figure 2 will be a helpful expansion of the tuning fork, serving as a reminder that some ETGs also have bars, and that they possess a range of disc sizes. The grid does not attempt to be all-encompassing. Not included are dwarf spheroidal galaxies (van den Bergh 1959), early- and late-type ultra diffuse galaxies with low surface brightnesses and diameters of $\sim 10 \mathrm{kpc}$ (Sandage \& Binggeli 1984), rare (less than a few percent at $z=0$ ) Irregular galaxies, nor interacting galaxies (Vorontsov-Velyaminov 1977). Rather than tack the latter two classes on at the end of the grid, the Irregular and Peculiar galaxies are considered here to be unrelaxed disturbed systems which are yet to settle into one of the types shown on the grid.

\section{DISCUSSION}

It has often been repeated over the last century that the merit of a galaxy classification scheme can be measured by its ability to track evolutionary pathways. As noted earlier, the changing, luminosity-weighted, mean age of the stellar population along the Jeans-Lundmark-Hubble sequence was routinely heralded as a success of this classification scheme. We now know that this suggestion by Sandage (1961), and others before him, that this agesequence might reflect an evolutionary pathway from right to left along the tuning fork, was not correct. However, a galaxy classification scheme has an additional benefit: it creates familiarity with the morphology, structure, and components that galaxies are comprised of.

It is hoped that the galaxy morphology classification grid shown in Figure 2 will raise awareness as to the presence and range of disc sizes in early-type galaxies. For example, Savorgnan \& Graham (2016b) reported on how a lack of awareness of intermediatescale discs led to claims of over-massive black holes in ES galaxies reported to have unusually high $M_{\mathrm{bh}} / M_{\text {bulge }}$ ratios. However, some of the galaxies had previously been modelled with large-scale discs, resulting in an underestimation of the bulge light.

To give another example, over the past decade, many clever studies have tried to understand how practically all compact massive spheroids at $z \sim 2.0 \pm 0.5$ could grow their 3D spheroid to become elliptical galaxies (e.g. Hopkins et al. 2009; Hilz et al. 2013; Oogi \& Habe 2013, and references therein). However, the similar sizes, masses, densities, Sérsic indices of local $(z=0)$ bulges and high- $z$ compact massive galaxies suggests an alternate solution to the fate of some of the high- $z$ compact massive galaxies which may have accreted/formed a disc rather than turned into an elliptical galaxy (Graham et al. 2015). For lenticular galaxies, their 2D discs can dramatically increase the size of the galaxy beyond the size of their 3D bulge. The stellar density within lenticular galaxy half light radii are less than the stellar density within the half light radii of their bulge components because bulges naturally pack in more stars by filling the $3 \mathrm{D}$ volume while discs only occupy a thickened plane. As shown in Graham (2013, his Figure 1), the density of bulges is higher than the density of ETGs of the same mass, except at the high-mass end where the ETGs are 3D spheroidal stellar systems. 


\subsection{Formation paths}

ETGs are often said to constitute the endpoint of galaxy evolution, such that they have exhausted their gas supply from which stars are formed, making them "red and dead". However, this picture is somewhat limited given the "down-sizing" nature to galaxy growth, in which lower-mass field galaxies form from smaller overdensities in the early Universe, and take longer to accrete their neighbouring gas supply. Given their smaller masses, significant fractions of material can still swoop in and continue to build these galaxies today. Indeed, the dwarf early-type galaxy CG 611 has young spiral arms in its intermediate-scale stellar disc that is undergoing growth via gas accretion in counter rotation to the stellar disc (Graham et al. 2017). The past growth of discs via accretion and minor mergers is also evident in more massive ETGs, with counter rotation seen in, for example, NGC 7007 and NGC 4550 (Dettmar et al. 1990; Rix et al. 1992). Therefore, the popular notion that gas poor ETGs with discs evolved from gas rich spiral galaxies is probably not the full picture. Rather, the accretional growth of discs building ES and S0 galaxies may be a fundamental pathway for galaxy growth over the past ten billion years.

Perhaps a key limitation of the classification of galaxies in their "ground state", and thus a preference for the interaction types of the MCG (Vorontsov-Vel'Yaminov \& Arkhipova 1962-1974) and the asymmetry and clumpiness indices, is that these may better reveal a progression or sequence of galaxies traversing the "excited states" to reach the "ground state" configuration. The hope of using the Jeans-Hubble tuning fork as a tool for understanding galaxy evolution, in a manner akin to how the HertzsprungRussell (HR) diagram (Rosenberg 1910; Hertzsprung 1911; Russell 1914; Nielsen 1964) is used for understanding stellar evolution, may have therefore been partly hamstrung from the get go. This was suspected at least as far back as Shapley \& Paraskevopoulos (1940) who wrote, in regard to the $1000+$ classified galaxies (e.g. Shapley \& Ames 1932), that: "The [rare] unusual forms, whether chaotically irregular ... or merely peculiar variations on the usual types ... [are] of uncommon interest and perhaps of special importance in the study of galactic structures and development." As such, the largely overlooked classification scheme for galaxy interactions (Vorontsov-Vel'Yaminov 1959) may be where one should look for evolutionary pathways. In a sense, while the HR diagram sampled stars in different phases of their evolution, perhaps the Jeans-Hubble diagram, with its emphasis on big evolved galaxies, in essence samples galaxies at a single (evolved) epoch. Much of the evolution may effectively be hidden in the connection between the now $(z=0)$ rare Irregular galaxies and the common structured galaxies, at odds with the (previously) expected notion of evolution between the structured galaxies. This is not to say that the latter does not occur. Indeed, disc accretion has likely converted some elliptical galaxies into ellicular and lenticular galaxies (Graham et al. 2015), which would represent limited evolution from left to right along a section of the the Jeans-Lundmark-Hubble sequence, as Jeans first speculated, but due to the accretion of external material rather than due to cooling, contraction, and spinning-up of an isolated nebula.

The grid presented in Figure 2 recognises the existence of bars in ETGs, as does de Vaucouleurs (1959a) three-dimensional classification volume and the Hubble-Sandage classification scheme (Sandage 1961), and it also captures the broader range of sizes of the spiral-less discs in ETGs that was first recognised by Liller (1966). It is noted, however, that ETGs, and in particular ES and S0 galaxies, are not limited to being three-component bulge+disc+bar systems (e.g. Cheng et al. 2011). Building upon the practice of simultaneous multi-component decompositions (e.g. de Jong 1996; Prieto et al. 1997, 2001), ETGs not only have bars within discs (e.g. Friedli \& Martinet 1993; Laurikainen et al. 2005), but they may also possess barlenses / (peanut shell)-shaped pseudobulges (Combes et al. 1990; Athanassoula 2005; Athanassoula et al. 2015; Laurikainen et al. 2011, 2018; Saha et al. 2018), ansae (e.g. Martinez-Valpuesta et al. 2007; Saha et al. 2018), nuclear-rings, bar-rings and outerrings (Theys \& Spiegel 1976; de Vaucouleurs et al. 1991; Michard \& Marchal 1993; Buta \& Combes 1996; Buta 2011; Mirtadjieva \& Nuritdinov 2016; Buta 2017), and nuclear stellar discs (e.g. Scorza \& Bender 1995; Scorza et al. 1998; Morelli et al. 2004; Balcells et al. 2007) which likely form a continuum with the intermediatescale discs of the ES galaxies and the large-scale discs of the S0 galaxies. This variety of components found within ETGs can be appreciated from the multi-component decompositions by Buta et al. (2010), Läsker et al. (2014), Savorgnan \& Graham (2016a), Davis et al. (2019), and Sahu et al. (2019).

Detailed information about the spiral arms in LTGs (e.g. Elmegreen \& Elmegreen 1982) may require higher-dimensions than offered by a two-dimensional schematic, although see Block \& Puerari (1999) and Seigar et al. (2005) in regard to use of spiral arm number and pitch angle in the near-infrared. As noted earlier, such higher-order information from more complex schemes can always be annotated to a primary classification, as done with the de Vaucouleurs volume.

\subsection{Kinematic classifications}

Although this study has focussed on the morphological classification of galaxies, it is insightful to review some of the relevant developments pursuing a kinematic classification. As noted previously, the prevalence of discs in ETGs has taken a remarkably long time to be realised. Indeed, most, if not all, relaxed E4 to E6/E7 galaxies are misclassified S0 galaxies (Liller 1966; Gorbachev 1970; Michard 1984; Capaccioli et al. 1990; van den Bergh 1990). Although many papers have pointed to the existence and abundance of discs in ETGs (e.g. Bertola \& Capaccioli 1975; Davies et al. 1983; Capaccioli 1987; Carter 1987; Nieto et al. 1988, 1991; Rix \& White 1990; Sandage \& Bedke 1994; Scorza \& Bender 1995; Graham et al. 1998; Rix et al. 1999; Krajnović et al. 2013; Scott et al. 2014; Bassett et al. 2017), in some quarters they are still not widely recognised, nor fully appreciated. However, this seems set to change with the increased amount of kinematic data becoming available.

After reporting, for several years, on the missed discs in ETGs, Capaccioli \& Caon (1992) wrote that "It was in fact possible to catalog ellipticals into two kinematical families: one dominated by random motions and made up of the most luminous galaxies, and another containing fainter objects characterized by a fair balance between random and ordered motions, just as in [the] bulges of S0 and S galaxies" and that the ETGs with discs describe "a sequence primarily described by the value of the specific angular momentum". ${ }^{23}$ Indeed, this is the basis of the kinematic classification which the SAURON project (Bacon et al. 2001; de Zeeuw et al.

23 This is somewhat reminiscent of the work by Alexander (1852), in which the angular momentum of the collapsing nebula was the determining factor in the production of elliptical or spiral nebulae; see also Shaya \& Tully (1984). 
2002) used to separate ETGs into slow or fast rotators (Emsellem et al. 2007).

Of course, what the SAURON project were in essence doing was re-labelling the E galaxies as "slow rotators" (SR), and the S0 and ES galaxies as "fast rotators" (FR). The strength in their approach was the ease at which they could detect discs in their kinematic data (including line-of-sight velocity $V$, and velocity dispersion $\sigma$ ) due to the $\sin (i)$, rather than $\cos (i)$, dependence on the inclination of the disc, as noted in Section 3.5. Furthermore, analysis of the shape of the absorption lines, and their deviations from a Gaussian, offered additional means for probing the embedded discs (Krajnović et al. 2008). Additional observations using the SAURON integral-field spectrograph on the William Herschel Telescope spectroscopically confirmed an abundance of discs in ETGs (Emsellem et al. 2011; Cappellari et al. 2011). The ATLAS ${ }^{3 D}$ team subsequently explored the bulge+disc nature of these ETGs through bulge+disc decompositions (Krajnović et al. 2013), and the flattened nature of these discs, as opposed to rotating ellipsoids, was shown through their study of the galaxy's axial ratios (Weijmans et al. 2014), building on Freeman (1970) and Sandage et al. (1970).

Considering ETGs to be single-component ellipsoids that can be flattened by either rotation or anisotropy in their velocity dispersion, Binney (1976, 1978, 1985; see also de Zeeuw \& Franx 1991) established a dividing line between fast and slow rotators in the ellipticity- $\left(V_{\max } / \sigma_{0}\right)$ diagram. Capellari et al. (2007) used this diagram to argue that the fast rotators are nearly oblate and contain disc-like components. Emsellem et al. (2007, see their Figure 5) introduced a clever variation by deriving the aperture "spin" parameter, $\lambda_{R}$, which replaced $V_{\max } / \sigma_{0}$, and where the subscript $R$ denoted the radius of the aperture. This $\epsilon-\lambda_{R}$ diagram has been used to classify galaxies, including ETGs, as either an FR or SR.

However, this FR/SR classification suffers from one of the problems that undermined the use of isophotes to classify ETGs as either boxy or discy. Specifically, unlike with spiral galaxies, for ETGs the presence of an inner disc does not ensure the presence of a disc at large radii. The FR versus SR designation has therefore propagated the tendency for one to overlook the ES galaxies which are both fast rotators at small radii and slow rotators at large radii. ${ }^{24}$ To help rectify this situation, Graham et al. (2017) suggested a further variation to the ellipticity-kinematic diagrams such that galaxies are not represented by a single aperture value but rather annular information is used to show the radially-varying behavior of their kinematics and ellipticity. Bellstedt et al. (2017) further revealed how incorporating the radial kinematic and photometric information aids in the identification of intermediate-scale discs, and counter-rotating discs, with galaxies moving along "radial tracks" in the modified spin-ellipticity $(\epsilon(R)-\lambda(R))$ diagram.

Expanding upon Krajnović et al. (2006), van de Sande et al. (2017) also kinematically classified galaxies, introducing five classes using the velocity dispersion, rotation, skewness and kurtosis of the stellar absorption lines, albeit using a single measure for each of these quantities within one effective half light radius, $R_{\mathrm{e}}$. When the "integral field spectrograph" data allows it, it may be additionally fruitful to represent the array of galaxy-wide information using more than one aperture radius (e.g. Chung \& Bureau 2002). Based on the 2D kinematic maps, Krajnović et al. (2011) divided the regular and non-regular rotators into seven subgroups:

24 Emsellem et al. (2007, see their Figure 2) were aware of this population, but the FR/SR convention that they used does not encapsulate it. featureless; low-rotation; kinematic twist; kinematically decoupled cores; counter rotating cores; double maxima in the radial velocity profile (2M galaxies); double maxima in the velocity dispersion map, i.e. $2 \sigma$ peaks. Most recently, Rawlings et al. (2019) have attempted to condense this $2 \mathrm{D}$ information via a suite of template radial tracks in the modified spin-ellipticity diagram for seven different morphological-dynamical types of galaxy, including the ES galaxies with intermediate-scale discs, the " $2 \sigma$ peak" galaxies, plus E, S0, early- and late-type spiral galaxies, and barred spiral galaxies.

\subsection{The future with Big Data sets}

The Universe is big. The observable portion of the Universe may contain in excess of $10^{11}$ galaxies, notably higher than the, at the time impressive, figure of nearly one million galaxies circa 1930. The dramatic increase in galaxy sample sizes since the early 1900s has resulted in an explosion of data and catalogs that all too often is not matched by the human resources required to fully analyse it. This inability to classify every galaxy using the ways of the past, i.e. visual inspection, could potentially undermine the current classification scheme. Indeed, there have been calls to replace galaxy morphological types with quantitative metrics that machines can quickly calculate. This final subsection serves to acknowledge the merits of such a suggestion based on robust non-degenerate metrics, and to briefly note how the community is moving forward on this front while still embracing key elements seen in both the tuning fork and the galaxy morphology classification grid.

Visual classification by a dedicated team of professionals (e.g. Vorontsov-Vel'Yaminov \& Arkhipova 1974; de Vaucouleurs et al. 1991; Driver et al. 2006; Fukugita et al. 2007; Buta et al. 2010; Nair \& Abraham 2010; Ann et al. 2015; Kartaltepe et al. 2015) has its limitations at around $10^{4}$ galaxies. As such, we have seen the birth of Citizen Scientist projects, with Galaxy Zoo recruiting up to $\sim 10^{5}$ budding and amateur astronomers who, collectively, can visually classify far more galaxies (e.g. Lintott et al. 2008; Willett et al. 2013; Dickinson et al. 2018). Furthermore, new branches of the galactic community are evolving through the exploration and application of software capable of semi-automatic galaxy classification on a large scale (e.g. Sedmak \& Lamas 1981; HuertasCompany et al. 2015). Examples of this Machine Learning (de la Calleja \& Fuentes 2004; Bazell \& Miller 2005; Shamir 2009; Barchi et al. 2019) include Support Vector Machines, statistical learning methods such as Classification Trees with Random Forest (CTRF) and Neural Networks (Huertas-Company et al. 2008; Diaz-Hernandez et al. 2016; Sreejith et al. 2018; Sultanova 2018), and enhanced brain storm optimization techniques (Ibrahim et al. 2018). Beck et al. (2018) describe how the preferably-large training sets for machine learning can effectively be provided by citizen scientists. Spectral (e.g. Morgan \& Mayall 1957; Sánchez Almeida et al. 2010; Wang et al. 2018) and kinematic (e.g. Brosche 1970; Wakamatsu 1976; Baiesi-Pillastrini 1987; Wiegert \& English 2014; Hung et al. 2015; van de Ven et al. 2016; Kalinova et al. 2017; van de Sande et al. 2017; Rawlings et al. 2019) information is additionally being folded into the mix, supplementing and complementing the morphological classification process.

\section{ACKNOWLEDGEMENTS}

It is a pleasure to thank the Department of Astronomy at The University of Florida, Gainesville, USA, for hosting me as a 
part of Swinburne University of Technology's Research Sabbatical Scheme. I am additionally grateful to James Josephides who converted my rough sketches into the Figures shown herein. This research was supported by Australian Research Council funding through grant DP110103509.

\section{REFERENCES}

Abraham, R. G., Valdes, F., Yee, H. K. C., \& van den Bergh, S. 1994, ApJ, 432,75

Abraham, R. G., Tanvir, N. R., Santiago, B. X., et al. 1996, MNRAS, 279, L47

Aitken, R. G. 1906, PASP, 18, 111

Alatalo, K., Davis, T. A., Bureau, M., et al. 2013, MNRAS, 432, 1796

Alexander, S. 1852, AJ, 2, 95

Ann, H. B., Seo, M., \& Ha, D. K. 2015, ApJS, 217, 27

Arnold, J. A., Romanowsky, A. J., Brodie, J. P., et al. 2014, ApJ, 791, 80

Athanassoula, E. 2002, ApJL, 569, L83

Athanassoula, E. 2003, MNRAS, 341, 1179

Athanassoula, E. 2005, MNRAS, 358, 1477

Athanassoula, E., Laurikainen, E., Salo, H., \& Bosma, A. 2015, MNRAS, 454, 3843

Bacon, R., Copin, Y., Monnet, G., et al. 2001, MNRAS, 326, 23

Baiesi-Pillastrini, G. C. 1987, A\&A, 172, 375

Baillard, A., Bertin, E., de Lapparent, V., et al. 2011, A\&A, 532, A74

Balcells, M., Graham, A. W., \& Peletier, R. F. 2007, ApJ, 665, 1084

Barchi, P. H., de Carvalho, R. R., Rosa, R. R., et al. 2019, Astronomy and Computing, submitted, arXiv:1901.07047

Bassett, R., Bekki, K., Cortese, L., et al. 2017, MNRAS, 470, 1991

Bazell, D., \& Miller, D. J. 2005, ApJ, 618, 723

Beck, M. R., Scarlata, C., Fortson, L. F., et al. 2018, MNRAS, 476, 5516

Begelman, M. C., Blandford, R. D., \& Rees, M. J. 1980, Nature, 287, 307

Bellstedt, S., Graham, A. W., Forbes, D. A., et al. 2017, MNRAS, 470, 1321

Bender, R. 1988, A\&A, 193, L7

Berentzen, I., Shlosman, I., \& Jogee, S. 2006, ApJ, 637, 582

Bertola, F., \& Capaccioli, M. 1975, ApJ, 200, 439

Bigay, J. H. 1963, L'Astronomie, 77, 173

Binney, J. 1976, MNRAS, 177, 19

Binney, J. 1978, MNRAS, 183, 501

Binney, J. 1985, MNRAS, 212, 767 de Vaucouleurs, G. 1981, MNRAS, 194, 679

Blakeslee, J. P., Holden, B. P., Franx, M., et al. 2006, ApJ, 644, 30

Block, D.L., Freeman, K.C. 2009, "Shrouds of the Night: Masks of the Milky Way and Our Awesome New View of Galaxies", New York, Springer, pp.441

Block, D. L., \& Puerari, I. 1999, A\&A, 342, 627

Block, D. L., Puerari, I., Freeman, K. C., Groess, R., \& Block, E. K. 2004, Penetrating Bars Through Masks of Cosmic Dust, Edited by D. L. Block, I. Puerari, K. C. Freeman, R. Groess, and E. K. Block. Astrophysics and space science library (ASSL) vol. 319. Dordrecht: Kluwer Academic Publishers, p.15-38

Boroson, T. 1981, ApJS, 46, 177

Brook, C. B., Kawata, D., Gibson, B. K., \& Freeman, K. C. 2004, ApJ, 612, 894

Brosche, P. 1970, A\&A, 6, 240

Burstein, D., Ho, L. C., Huchra, J. P., \& Macri, L. M. 2005, ApJ, 621, 246

Buta R. J., 2011, in Keel W. C., in "Planets, Stars and Stellar Systems", Volume 6, T.D.Oswalt \& W.C.Keel (Eds.), Springer-Verlag, Berlin

Buta, R. J. 2017, MNRAS, 471, 4027

Buta, R., \& Combes, F. 1996, Fundamentals of Cosmic Physics, 17, 95

Buta, R. J., Sheth, K., Regan, M., et al. 2010, ApJS, 190, 147

Campbell, W. W., \& Paddock, G. F. 1918, PASP, 30, 68

Caon, N., Capaccioli, M., \& D’Onofrio, M. 1993, MNRAS, 265, 1013

Capaccioli, M. 1987, Structure and Dynamics of Elliptical Galaxies, 127, 47
Capaccioli, M. 1990, European Southern Observatory Conference and Workshop Proceedings, 35, 231

Capaccioli, M., \& Caon, N. 1992, in Morphological and Physical Classification of Galaxies, Dordrecht: Kluwer Academic Publishers, 1992, edited by G. Longo, M. Capaccioli, and G. Busarello. Astrophysics and Space Science Library, Vol. 178, p.99

Capaccioli, M., Caon, N., \& Rampazzo, R. 1990, MNRAS, 242, 24P

Capaccioli, M., Piotto, G., \& Rampazzo, R. 1988, AJ, 96, 487

Capaccioli, M., \& Vietri, M. 1988, in Origin, Structure and Evolution of Galaxies, F.L. Zhi (ed), Singapore and Teaneck, NJ, World Scientific, p.150-161

Cappellari, M., Emsellem, E., Bacon, R., et al. 2007, MNRAS, 379, 418

Cappellari, M., Emsellem, E., Krajnović, D., et al. 2011, MNRAS, 416, 1680

Carter, D. 1978, MNRAS, 182, 797

Carter, D. 1987, ApJ, 312, 514

Cawson, M. 1983, in Astronomical Measuring Machines Workshop, Edinburgh Royal Observatory, Occasional Reports, R.O.E., 10, 175-184

Chamberlin, T. C. 1901, ApJ, 14, 17

Cheng, J. Y., Faber, S. M., Simard, L., et al. 2011, MNRAS, 412, 727

Chung, A., \& Bureau, M. 2002, Bulletin of the American Astronomical Society, vol.34, p.1116, id.13.12

Ciambur, B. C. 2015, ApJ, 810, 120

Combes, F., Debbasch, F., Friedli, D., \& Pfenniger, D. 1990, A\&A, 233, 82

Combes, F., \& Elmegreen, B. G. 1993, A\&A, 271, 391

Combes, F., \& Sanders, R. H. 1981, A\&A, 96, 164

Conselice, C. J. 1997, PASP, 109, 1251

Conselice, C. J. 2003, ApJS, 147, 1

Conselice, C. J. 2006, MNRAS, 373, 1389

Cortese, L. 2012, A\&A, 543, A132

Curtis, H. D. 1918, Publications of Lick Observatory, 13, 9

Curtis, H. D. 1933, Handbuch der Astrophysik, 5, 774

Danver, C.-G. 1942, Thesis, Annals of the Observatory of Lund, 10, pp.193

Davies, J. I., Phillipps, S., Cawson, M. G. M., Disney, M. J., \& Kibblewhite, E. J. 1988, MNRAS, 232, 239

Davies, R. L., Efstathiou, G., Fall, S. M., Illingworth, G., \& Schechter, P. L. 1983, ApJ, 266, 41

Davis, B. L., Graham, A. W., \& Seigar, M. S. 2017, MNRAS, 471, 2187

Davis, B. L., Graham, A. W., \& Cameron, E. 2019, ApJ, 873, 85

Debattista, V. P., \& Sellwood, J. A. 2000, ApJ, 543, 704

de Jong, R. S. 1996, A\&As, 118, 557

de la Calleja, J., \& Fuentes, O. 2004, MNRAS, 349, 87

Deng, X.-F., He, J.-Z., Wu, P., \& Ding, Y.-P. 2009, ApJ, 699, 948

Dettmar, R.-J., Jullien-Dettmar, M., \& Barteldrees, A. 1990, in The Interstellar Medium in External Galaxies, Proceedings of the Second Wyoming Conference, D.J. Hollenbach (ed.), NASA Conference Publication 3084, p. 246

di Tullio, G. 1978, A\&A, 62, L17

de Vaucouleurs, G. 1959a, Handbuch der Physik, 53, 275

de Vaucouleurs, G. 1959b, Handbuch der Physik, 53, 311

de Vaucouleurs, G. 1977, Evolution of Galaxies and Stellar Populations, eds. R. Larson \& B. Tinsley (New Haven: Yale University Observatory), p.43

de Vaucouleurs G., de Vaucouleurs A., Corwin H. G. J., Buta R. J., Paturel G., Fouque P., 1991, Third Reference Catalogue of Bright Galaxies, Springer

de Zeeuw, P. T., Bureau, M., Emsellem, E., et al. 2002, MNRAS, 329, 513

de Zeeuw, T., \& Franx, M. 1991, ARA\&A, 29, 239

Diaz-Hernandez, R., Ortiz-Esquivel, A., Peregrina-Barreto, H., AltamiranoRobles, L., \& Gonzalez-Bernal, J. 2016, Experimental Astronomy, 41, 409

Dickinson, H., Fortson, L., Lintott, C., et al. 2018, ApJ, 853, 194

Doi, M., Kashikawa, N., Okamura S., Tarusawa. K., Fukugita, M., Sekiguchi, M., \& Iwashita, H. 1992, in Digitised Optical Sky Surveys, Kluwer, Dordrecht, p.199

Doi, M., Fukugita, M., \& Okamura, S. 1993, MNRAS, 264, 832

Dreyer, J. L. E. 1888, A New General Catalogue of Nebulae and Clusters of Stars, Mem. R. Astron. Soc., 49, 1 
Dreyer, J. L. E. 1895, Index Catalogue of Nebulae found in the years 1888 to 1894, Mem. R. Astron. Soc., 51, 185

Driver, S. P., Allen, P. D., Graham, A. W., et al. 2006, MNRAS, 368, 414

Driver, S. P., Windhorst, R. A., \& Griffiths, R. E. 1995, ApJ, 453, 48

Dullo, B. T., \& Graham, A. W. 2014, MNRAS, 444, 2700

Elmegreen, D. M., \& Elmegreen, B. G. 1982, MNRAS, 201, 1021

Emsellem, E., Cappellari, M., Krajnović, D., et al. 2007, MNRAS, 379, 401

Emsellem, E., Cappellari, M., Krajnović, D., et al. 2011, MNRAS, 414, 888

Fath, E. A. 1909, Lick Observatory Bulletin, 5, 71

Feitzinger, J. V., \& Schmidt-Kaler, T. 1980, A\&A, 88, 41

Ferrarese, L., Côté, P., Jordán, A., et al. 2006, ApJS, 164, 334

Ferrari, F., de Carvalho, R. R., \& Trevisan, M. 2015, ApJ, 814, 55

Fiacconi, D., Feldmann, R., \& Mayer, L. 2015, MNRAS, 446, 1957

Foster, C., Pastorello, N., Roediger, J., et al. 2016, MNRAS, 457, 147

Fraser, C. W. 1972, The Observatory, 92, 51

Freeman, K. C. 1970, ApJ, 160, 811

Freeman, K. C. 1975, in Galaxies and the Universe, Edited by Allan Sandage, Mary Sandage, and Jerome Kristian, University of Chicago Press (Stars and Stellar Systems. Volume 9), Chicago, IL USA., 1975, p.409

Friedli, D., \& Martinet, L. 1993, A\&A, 277, 27

Fukugita, M., Nakamura, O., Okamura, S., et al. 2007, AJ, 134, 579

Genel, S., Nelson, D., Vogelsberger, M., et al. 2015, IAU General Assembly, 22, 2257577

George, K., \& Zingade, K. 2015, A\&A, 583, A103

Giavalisco, M., Livio, M., Bohlin, R. C., Macchetto, F. D., \& Stecher, T. P. 1996, AJ, 112, 369

Gorbachev, V. I. 1970, Soviet Astronomy, 14, 182 (Azh, 47, 224)

Gott, J. R., III 1975, ApJ, 201, 296

Graham, A. W. 2001, MNRAS, 326, 543

Graham, A. W. 2013, in "Planets, Stars and Stellar Systems", Volume 6, p.91-140, T.D.Oswalt \& W.C.Keel (Eds.), Springer Publishing (arXiv:1108.0997)

Graham, A. W. 2019, PASA, in press

Graham, A. W., Colless, M. M., Busarello, G., Zaggia, S., \& Longo, G. 1998, A\&A Supp., 133, 325

Graham, A. W., Ciambur, B. C., \& Savorgnan, G. A. D. 2016, ApJ, 831, 132

Graham, A. W., \& Driver, S. P. 2005, PASA, 22, 118

Graham, A. W., Dullo, B. T., \& Savorgnan, G. A. D. 2015, ApJ, 804, 32

Graham, A. W., Erwin, P., Trujillo, I., \& Asensio Ramos, A. 2003, AJ, 125, 2951

Graham, A. W., Janz, J., Penny, S. J., et al. 2017, ApJ, 840, 68

Graham, A. W., Spitler, L. R., Forbes, D. A., et al. 2012, ApJ, 750, 121

Graham, A. W., Trujillo, I., \& Caon, N. 2001, AJ, 122, 1707

Graham, A. W., \& Worley, C. C. 2008, MNRAS, 388, 1708

Griffiths, R. E., Casertano, S., Ratnatunga, K. U., et al. 1994, ApJL, 435, L19

Groot, H. 1925, MNRAS, 85, 535

Gurevich, L. E., \& Lebedinsky, A. I. 1950, Izvestia Academii Nauk, USSR, 14,765

Gutiérrez, L., Erwin, P., Aladro, R., \& Beckman, J. E. 2011, AJ, 142, 145

Hart, R., \& Berendzen, R. 1971, Journal for the History of Astronomy, 2, 109

Haass, J., Bertin, G., \& Lin, C. C. 1982, Proceedings of the National Academy of Science, 79, 3908

Herschel, W. 1786, Philosophical Transactions of the Royal Society of London Series I, 76, 457

Herschel, J. F. W. 1833, Philosophical Transactions of the Royal Society of London Series I, 123, 359

Herschel, J. 1864, General Catalogue of Nebulae and Clusters of Stars, Royal Society of London Philosophical Transactions Series I, 154, 1

Hertzsprung, E. 1911, Publikationen des Astrophysikalischen Observatoriums zu Potsdam, 22. Bd., 1. Stuck = Nr. 63

Hilz, M., Naab, T., \& Ostriker, J. P. 2013, MNRAS, 429, 2924

Hodge, P. W. 1966, McGraw-Hill Series in Undergraduate Astronomy, The Physics and Astronomy of Galaxies and Cosmology. McGraw-Hill, New York
Hohl, F. 1971, ApJ, 168, 343

Holmberg, E. 1958, Meddelanden fran Lunds Astronomiska Observatorium Serie II, 136, 1

Hopkins, P. F., Hernquist, L., Cox, T. J., Keres, D., \& Wuyts, S. 2009, ApJ, 691,1424

Hubble, E. P. 1922, ApJ, 56, 162

Hubble, E. 1923, Popular Astronomy, 31, 644

Hubble, E. P. 1926, ApJ, 64, 321

Hubble, E. 1929, Proceedings of the National Academy of Science, 15, 168

Hubble, E.P. 1936, Realm of the Nebulae, by E.P. Hubble, New Haven: Yale University Press

Huertas-Company, M., Gravet, R., Cabrera-Vives, G., et al. 2015, ApJS, 221,8

Huertas-Company, M., Rouan, D., Tasca, L., Soucail, G., \& Le Fèvre, O. 2008, A\&A, 478, 971

Humason, M. L., Mayall, N. U., \& Sandage, A. R. 1956, AJ, 61, 97

Hung, C.-L., Rich, J. A., Yuan, T., et al. 2015, ApJ, 803, 62

Hunter, C. 1963, MNRAS, 126, 299

Ibrahim, R. A., Elaziz, M. A., Ewees, A. A., Selim, I. M., \& Lu, S. 2018, Journal of Astronomical Telescopes, Instruments, and Systems, 4, 038001

Isserstedt, J., \& Schindler, R. 1986, A\&A, 167, 11

Jacobi, C.G. 1834, Annalen der Physik, vol.109, Issue 8, p.229

Janz, J., Penny, S. J., Graham, A. W., Forbes, D. A., \& Davies, R. L. 2017 , MNRAS, 468, 2850

Jarrett, T. H., Chester, T., Cutri, R., Schneider, S. E., \& Huchra, J. P. 2003, AJ, 125, 525

Jeans, J. H. 1902, Philosophical Transactions of the Royal Society of London Series A, 199, 1

Jeans J. 1919a. Problems of Cosmogony and Stellar Dynamics, Cambridge: Cambridge Univ. Press

Jeans, J. H. 1919b, Journal of the Royal Astronomical Society of Canada, 13,215

Jeans, J.H. 1928, Astronomy \& Cosmogony, (Cambridge: Cambridge University Press), p.332

Jeans, J.H. 1931, “An evolving Universe”, Carnegie Institution of Washington, News Service Bulletin, Staff Edition, No.23, p.157

Jerjen, H., \& Binggeli, B. 1997, The Nature of Elliptical Galaxies; 2nd Stromlo Symposium, 116, 239

Kalinova, V., Colombo, D., Rosolowsky, E., et al. 2017, MNRAS, 469, 2539

Kannappan, S. J., Guie, J. M., \& Baker, A. J. 2009, AJ, 138, 579

Kant, I. 1755, Allgemeine Naturgeschichte und Theorie des Himmels, Germany, Universal Natural History and Theory of the Heavens, p.367; translated by Stephen Palmquist in Kant's Critical Religion (Aldershot: Ashgate, 2000), pp.320

Kartaltepe, J. S., Mozena, M., Kocevski, D., et al. 2015, ApJS, 221, 11

Kaviraj, S., Tan, K.-M., Ellis, R. S., \& Silk, J. 2011, MNRAS, 411, 2148

Keeler, J. E. 1900, ApJ, 11, 325

Kent, S. M. 1985, ApJS, 59, 115

Knox-Shaw, H. 1915, Helwan Institute of Astronomy and Geophysics Bulletins, 15,129

Kodaira, K., Watanabe, M., \& Okamura, S. 1986, ApJS, 62, 703

Kormendy, J., \& Bender, R. 1996, ApJL, 464, L119

Kormendy, J., \& Bender, R. 2009, ApJL, 691, L142

Kormendy, J., \& Bender, R. 2012, ApJS, 198, 2

Krajnović, D., Alatalo, K., Blitz, L., et al. 2013, MNRAS, 432, 1768

Krajnović, D., Bacon, R., Cappellari, M., et al. 2008, MNRAS, 390, 93

Krajnović, D., Cappellari, M., de Zeeuw, P. T., \& Copin, Y. 2006, MNRAS, 366, 787

Krajnović, D., Emsellem, E., Cappellari, M., et al. 2011, MNRAS, 414, 2923

de La Caille, N.L., 1755, Sur les étoiles nébuleuses du Ciel Austral [On the nebulous stars of the Southern Sky]. Memoirs of the Royal Academy for 1755 (Paris), pp.194-199

Lahav, O., Naim, A., Buta, R. J., et al. 1995, Science, 267, 859

Laplace, P.S. 1796, Exposition du Systéme du monde, (1809 English translation)

Laplace, P.S. 1799, 1802, 1805, 1825, Mécanique Céleste, Volumes 1-5 
Larson, R. B. 1975, MNRAS, 173, 671

Läsker, R., Ferrarese, L., \& van de Ven, G. 2014, ApJ, 780, 69

Laurikainen, E., Salo, H., \& Buta, R. 2005, MNRAS, 362, 1319

Laurikainen, E., Salo, H., Buta, R., Knapen, J. H., \& Comerón, S. 2010, MNRAS, 405, 1089

Laurikainen, E., Salo, H., Buta, R., \& Knapen, J. H. 2011, MNRAS, 418, 1452

Laurikainen, E., Salo, H., Laine, J., \& Janz, J. 2018, A\&A, 618, A34

Lee, J. H., Lee, M. G., \& Hwang, H. S. 2006, ApJ, 650, 148

Lekshmi, S., Revathy, K., \& Prabhakaran Nayar, S. R. 2003, A\&A, 405, 1163

Liller M. H., 1966, ApJ, 146, 28

Lindblad, B., \& Elvius, A. 1952, Nature, 169, 1042

Lintott, C. J., Schawinski, K., Slosar, A., et al. 2008, MNRAS, 389, 1179

Lotz, J. M., Primack, J., \& Madau, P. 2004, AJ, 128, 163

Lundmark, K. 1925, MNRAS, 85, 865

Lundmark, K. 1927, Nova Acta Regiae Soc. Sci. Upsaliensis, Volumen Extra Ordinem Editum, Ser. V, 1, pp.127

MacArthur, L.A., González, J.J., Courteau, S. 2009, MNRAS, 395, 28

Maclaurin, C.A. 1742, Treatise of Fluxions, Vol. 1., Edinburgh: Printed by T.W. and T. Ruddimans

Mahajan, S., Drinkwater, M. J., Driver, S., et al. 2018, MNRAS, 475, 788

Martinez-Valpuesta, I., Knapen, J. H., \& Buta, R. 2007, AJ, 134, 1863

Masters, K. L., Mosleh, M., Romer, A. K., et al. 2010, MNRAS, 405, 783

Matthews, T. A., Morgan, W. W., \& Schmidt, M. 1964, ApJ, 140, 35

Mei, S., Blakeslee, J. P., Stanford, S. A., et al. 2006, ApJ, 639, 81

Messier, C. 1781, Connoissance des Temps ou des Mouvements Célestes, for 1784, p. $227-267$

Michard, R. 1984, A\&A, 140, L39

Michard, R., \& Marchal, J. 1993, A\&As, 98, 29

Michard, R., \& Simien, F. 1988, A\&A Supp., 74, 25

Mirtadjieva, K. T., \& Nuritdinov, S. N. 2016, Astronomical and Astrophysical Transactions, 29, 305

Moffett, A. J. 2014, PhD Thesis, The University of North Carolina at Chapel Hill

Morelli, L., Halliday, C., Corsini, E. M., et al. 2004, MNRAS, 354, 753

Morgan, W. W. 1958, PASP, 70, 364

Morgan, W. W. 1959, PASP, 71, 394

Morgan, W. W., \& Lesh, J. R. 1965, ApJ, 142, 1364

Morgan, W. W., \& Mayall, N. U. 1957, PASP, 69, 291

Morgan, W. W., \& Osterbrock, D. E. 1969, AJ, 74, 515

Mortlock, A., Conselice, C. J., Hartley, W. G., et al. 2013, MNRAS, 433, 1185

Moulton, F. R. 1905, ApJ, 22, 165

Nair, P. B., \& Abraham, R. G. 2010, ApJS, 186, 427

Neichel, B., Hammer, F., Puech, M., et al. 2008, A\&A, 484, 159

Nielsen, A. V. 1964, Centaurus, 9, 219

Nieto, J.-L., Capaccioli, M., \& Held, E. V. 1988, A\&A, 195, L1

Nieto, J.-L., Poulain, P., Davoust, E., \& Rosenblatt, P. 1991, A\&A Supp., 88,559

Okamura, S., Kodaira, K., \& Watanabe, M. 1984, ApJ, 280, 7

Oogi, T., \& Habe, A. 2013, MNRAS, 428, 641

Parson L. 1878, Scientific Transactions of the Royal Dublin Society Vol. II

Pović, M., Márquez, I., Masegosa, J., et al. 2015, MNRAS, 453, 1644

Prentice, A. J. R. 1978, Moon and Planets, 19, 341

Prentice, A. J. R. 1984, Earth Moon and Planets, 30, 209

Prentice, A. J. R., \& Dyt, C. P. 2003, MNRAS, 341, 644

Prieto, M., Aguerri, J. A. L., Varela, A. M., \& Muñoz-Tuñón, C. 2001, A\&A, 367, 405

Prieto, M., Gottesman, S. T., Aguerri, J.-A. L., \& Varela, A.-M. 1997, AJ, 114,1413

Rawlings, A., Foster, C., and the SAMI team, 2019, MNRAS, submitted

Remus, R.-S., Dolag, K., Bachmann, L. K., et al. 2015, Galaxies in 3D across the Universe, Proc. IAU Symp. 309, 145

Reynolds, J. H. 1913, MNRAS, 74, 132

Reynolds, J. H. 1916, The Observatory, 39, 174

Reynolds, J. H. 1920, MNRAS, 80, 746

Reynolds, J. H. 1921, The Observatory, 44, 368
Reynolds, J. H. 1925, MNRAS, 85, 1014

Reynolds, J. H. 1927, The Observatory, 50, 185

Rix, H.-W., Carollo, C. M., \& Freeman, K. 1999, ApJL, 513, L25

Rix, H.-W., Franx, M., Fisher, D., \& Illingworth, G. 1992, ApJL, 400, L5

Rix, H.-W., \& White, S. D. M. 1990, ApJ, 362, 52

Rix, H.-W., \& Zaritsky, D. 1995, ApJ, 447, 82

Roberts, W. W., Jr., Roberts, M. S., \& Shu, F. H. 1975, ApJ, 196, 381

Roche, E. 1850, Mémoires divers sur l'équilibre d'une masse fluide, Acadámie de Montpellier. Mámoires de la section des sciences, p.333

Rood, H. J., \& Baum, W. A. 1967, AJ, 72, 398

Rosenberg, H. 1910, Astronomische Nachrichten, 186, 71

Rosse, T. E. O. 1850a, Philosophical Transactions of the Royal Society of London Series I, 140, 499

Rosse, T. E. O. 1850b, MNRAS, 10, 21

Russell, H. N. 1914, Popular Astronomy, 22, 275

Safronov, V. S. 1960, Annales d'Astrophysique, 23, 979

Safronov, V. 1969, Evolution of the Protoplanetary Cloud and Formation of the Earth and the Planets. Moscow: Nauka Press, Trans. NASA TTF 677, 1972.

Saha, K., Graham, A. W., \& Rodríguez-Herranz, I. 2018, ApJ, 852, 133

Sahu, N., Graham, A. W., \& Davis, B. L. 2019, ApJ, in press, arXiv: 1903.04738

Sánchez Almeida, J., Aguerri, J. A. L., Muñoz-Tuñón, C., \& de Vicente, A. 2010, ApJ, 714, 487

Sandage, A. 1961, The Hubble Atlas of Galaxies (Carnegie Institution of Washington, Washington, D.C.)

Sandage, A. 1975, Galaxies and the Universe, Stars and Stellar Systems. Vol. 9, University of Chicago Press, p.1

Sandage, A., \& Bedke, J. 1994, The Carnegie Atlas of Galaxies. Volumes I, II., by Sandage, A.; Bedke, J.. Carnegie Institution of Washington Publ., No. 638,

Sandage, A., \& Binggeli, B. 1984, AJ, 89, 919

Sandage, A., Freeman, K. C., \& Stokes, N. R. 1970, ApJ, 160, 831

Savorgnan, G. A. D., \& Graham, A. W. 2016a, ApJS, 222, 10

Savorgnan, G. A. D., \& Graham, A. W. 2016b, MNRAS, 457, 320

Scarlata, C., Carollo, C. M., Lilly, S., et al. 2007, ApJS, 172, 406

Schade, D., Lilly, S. J., Crampton, D., et al. 1995, ApJL, 451, L1

Schawinski, K., Lintott, C., Thomas, D., et al. 2009, MNRAS, 396, 818

Schwarzschild, K. 1913, ApJ, 37, 294

Scorza, C., \& Bender, R. 1995, A\&A, 293, 20

Scorza, C., Bender, R., Winkelmann, C., Capaccioli, M., \& Macchetto, D. F. 1998, A\&As, 131, 265

Scott, N., Davies, R. L., Houghton, R. C. W., et al. 2014, MNRAS, 441, 274

Sedmak, G., \& Lamas, M. L. T. 1981, A\&A, 104, 93

Seigar, M. S., Block, D. L., Puerari, I., Chorney, N. E., \& James, P. A. 2005, MNRAS, 359, 1065

Selim, I. M., \& Abd El Aziz, M. 2017, Experimental Astronomy, 43, 131

Sellwood, J. A., \& Wilkinson, A. 1993, Reports on Progress in Physics, 56, 173

Sérsic, J. L. 1963, Boletin de la Asociacion Argentina de Astronomia La Plata Argentina, 6, 41

Shamir, L. 2009, MNRAS, 399, 1367

Shapley, H. 1927, Harvard College Observatory Bulletin, 849, 1

Shapley, H., \& Ames, A. 1929, Harvard College Observatory Bulletin, 866, 20

Shapley, H., \& Ames, A. 1932, Annals of Harvard College Observatory, 88, 41

Shapley, H., \& Paraskevopoulos, J. S. 1940, Proceedings of the National Academy of Science, 26, 31

Shaya, E. J., \& Tully, R. B. 1984, ApJ, 281, 56

Simien, F., \& de Vaucouleurs, G. 1986, ApJ, 302, 564

Simien, F., \& Michard, R. 1990, A\&A, 227, 11

Slipher, V. M. 1915, Popular Astronomy, 23, 21

Slipher, V. M. 1917, Proceedings of the American Philosophical Society, 56,403

Spitzer, L., Jr., \& Baade, W. 1951, ApJ, 113, 413

Sreejith, S., Pereverzyev, S., Jr., Kelvin, L. S., et al. 2018, MNRAS, 474, 5232 
Sultanova, M. R. 2018, Ph.D. Thesis, The University of North Dakota, Dissertation Abstracts International, Volume: 80-02(E), Section: B.; 207 Sutherland, W. 1911, ApJ, 34, 251

Swedenborg, E. 1734, Princlpia rerum naturalium sive novorum tentaminium phenomena mundi elementaris philosophice explicandi. Dresden and Leipzig.

Takamiya, M. 1999, ApJS, 122, 109

Teerikorpi, P. 1989, Journal for the History of Astronomy, 20, 165

Theys, J. C., \& Spiegel, E. A. 1976, ApJ, 208, 650

Tisserand, F. 1889-1896, Traité de mécanique céleste

Toomre, A. 1964, ApJ, 139, 1217

Toomre, A. 1981, Structure and Evolution of Normal Galaxies, Fall, S.M. and Lynden-Bell, D., eds, 111

Trujillo, I., Graham, A. W., \& Caon, N. 2001, MNRAS, 326, 869

Valentini, M., Murante, G., Borgani, S., et al. 2017, MNRAS, 470, 3167

van de Sande, J., Bland-Hawthorn, J., Fogarty, L. M. R., et al. 2017, ApJ, 835,104

van de Ven, G., Zhu, L., van den Bosch, R. C. E., et al. 2016, The Interplay between Local and Global Processes in Galaxies, 25

van den Bergh, S. 1959, Publications of the David Dunlap Observatory, 2, 147

van den Bergh, S. 1960a, Publications of the David Dunlap Observatory, 2, 159

van den Bergh, S. 1960b, ApJ, 131, 215

van den Bergh, S. 1960c, ApJ, 131, 558

van den Bergh, S. 1966, AJ, 71, 922

van den Bergh, S. 1975, Journal of the Royal Astronomical Society of Canada, 69, 57

van den Bergh, S. 1976, ApJ, 206, 883

van den Bergh, S. 1990, ApJ, 348, 57

Vika, M., Vulcani, B., Bamford, S. P., Häußler, B., \& Rojas, A. L. 2015, A\&A, 577, A97

von der Pahlen, E. 1911, Astronomische Nachrichten, 188, 249

Vorontsov-Vel'Yaminov, B. A. 1959, Atlas and catalog of interacting galaxies, Vol. 1., Sternberg Institute, Moscow

Vorontsov-Velyaminov, B. A. 1977, A\&A Supp., 28, 1

Vorontsov-Vel'Yaminov, B. A., \& Arkhipova, V. P. 1962-1968, Morphological catalogue of galaxies, Vol.1-4, State Univ. Moscow

Vorontsov-Vel'Yaminov, B. A., \& Arkhipova, V. P. 1974, Trudy Gosudarstvennogo Astronomicheskogo Instituta, 46,

Vorontsov-Vel'Yaminov, B. A., \& Noskova, R. I. 1968, Soviet Astronomy, 12,112

Wakamatsu, K.-I. 1976, PASJ, 28, 397

Walcher, C. J., van der Marel, R. P., McLaughlin, D., et al. 2005, ApJ, 618, 237

Wang, L.-L., Luo, A.-L., Shen, S.-Y., et al. 2018, MNRAS, 474, 1873

Wei, L. H., Kannappan, S. J., Vogel, S. N., \& Baker, A. J. 2010, ApJ, 708, 841

Weijmans, A.-M., de Zeeuw, P. T., Emsellem, E., et al. 2014, MNRAS, 444, 3340

Wiegert, T., \& English, J. 2014, New Astron., 26, 40

Willett, K. W., Lintott, C. J., Bamford, S. P., et al. 2013, MNRAS, 435, 2835

Wolf, M. 1908, Publikationen des Astrophysikalischen Instituts Koenigstuhl-Heidelberg, 3, 109

Yamauchi, C., Ichikawa, S.-i., Doi, M., et al. 2005, AJ, 130, 1545

Young, L., Bureau, M., Crocker, A., \& Combes, F. 2008, Formation and Evolution of Galaxy Bulges, 245, 173 